\title{
Numerical study of coupled-bunch instability caused by an electron cloud
}

\author{
S. S. Win, K. Ohmi, H. Fukuma, M. Tobiyama, J. Flanagan, and S. Kurokawa \\ KEK, 1-1 Oho, Tsukuba, 305-0801, Japan \\ (Received 14 March 2005; published 29 September 2005)
}

\begin{abstract}
An electron cloud induces a wake force on a charged particle beam which creates correlations between bunches - i.e., a small displacement of a bunch creates a perturbation of the electron cloud, which affects the motions of the following bunches, with the result that a coupled-bunch instability is caused. The coupling mode of the instability is determined by the motion of the electrons in the cloud-that is, it depends on which electrons, moving in a drift space, a weak solenoid field or a strong bending field, are dominant for the instability. We discuss the coupled-bunch instability focusing on the relation between the mode spectrum and the electron motion.
\end{abstract}

DOI: 10.1103/PhysRevSTAB.8.094401

PACS numbers: 29.20.Dh, 29.27. $-\mathrm{a}, 41.75 . \mathrm{Ht}$

\section{INTRODUCTION}

A positron or proton beam creates electrons due to synchrotron radiation, beam particle loss, and secondary emission due to the absorption of primary electrons at the vacuum chamber surface. In the case of multibunch operation with a short bunch spacing, electrons build up in the vacuum chamber by successive production, with the result that an electron cloud with a certain density is formed in the chamber. The beam passes through the cloud and interacts with it. The motions of bunches become correlated with each other if the memory of a previous bunch is retained in the electron cloud. When one bunch oscillates with a small betatron amplitude, other bunches are affected by the motion of the bunch via the electron cloud, with the result that a coupled-bunch instability is caused.

Transverse coupled-bunch instabilities caused by electron clouds have been observed in some positron storage rings: KEK-PF [1,2], CESR [3], and BEPC [4]. The instability has been also observed in the KEKB LER [5]. The mode spectrum, which characterizes unstable coupledbunch modes, has been measured with a fast beam-position monitor at KEKB. Many solenoid magnets were placed around the vacuum chambers around the the ring [6] to reduce single-bunch electron cloud effects [7-9]. An experiment performed at the KEKB LER showed that the solenoid magnets strongly affected the coupled-bunch instability, and a simulation followed the experimental results [5].

We discuss the coupled-bunch instability caused by electron clouds in drift spaces, weak solenoids and strong bending magnets using a (semi)analytic method based on the wake force and particle tracking simulation in this paper. It is shown that the electron motion, which is affected by the presence of magnetic fields, is reflected in the coupled-bunch instability. The results also tell us that the mode spectrum of the coupled-bunch instability indicates which electrons, in drift spaces, solenoid magnets, bending magnets or other magnets, are dominant in the actual ring.
A review of the mode frequency and spectrum of the coupled-bunch instability is presented in Sec. II. The physical interaction between the bunched beam and the electron cloud is reviewed in Sec. III. To analyze the coupled-bunch instability, a photoelectron cloud model has been used [2]. A wake force induced by the electron cloud is obtained in the model. The growth rate of each coupled-bunch mode is evaluated from the wake force in Sec. IV. The interaction between the bunch train and the electron cloud is also simulated by a tracking method in Sec. V. The tracking method gives the evolution of the transverse amplitudes of the bunches. The growth rate of each mode is obtained by a Fourier analysis of the amplitude evolution, and is compared with that given by the wake force. In Sec. VI, a longitudinal coupled-bunch instability due to electron cloud proposed by Novokhatski [10] is discussed.

We discuss the instability in the KEKB LER. Basic parameters of the KEKB LER are shown in Table I.

\section{MODE OF THE COUPLED-BUNCH INSTABILITY}

The coupled-bunch instability is a phenomenon wherein some coupled oscillation modes become unstable due to correlations between bunches; in this paper, the mediation of these correlations by electron clouds is discussed. The instability is characterized by the mode number $(m)$. We first consider the case where a train of $M$ bunches fills the machine circumference uniformly with equal spacing $\left(L_{\mathrm{sp}}=L / M\right)$. The transverse coordinate of a bunch $\left(y_{n}\right)$, denoted by $n$, is expressed by the mode number as

$$
y_{n, m}(t)=a_{m} \exp \left[-i \omega_{\beta} t+2 \pi i m n / M\right],
$$

where $a_{m}$ is the amplitude of the mode, a bunch with a larger value of $n$ is ahead of that with a smaller value of $n$, and $\omega_{\beta}$ and $M$ are the betatron frequency and the number of bunches, respectively.

We observe the coordinates of each bunch with a position monitor. The monitor gives the positions of bunches at 
TABLE I. Basic parameters of the KEKB LER.

\begin{tabular}{lcc}
\hline \hline \multicolumn{1}{c}{ Variable } & Symbol & KEKB LER \\
\hline Circumference & $L$ & $3016 \mathrm{~m}$ \\
Beam energy & $E$ & $3.5 \mathrm{GeV}$ \\
Bunch population & $N_{p}$ & $5.0 \times 10^{10}$ \\
Bunch spacing & $t_{\mathrm{sp}}=L_{\mathrm{sp}} / c$ & $2-8 \mathrm{~ns}$ \\
rms beam sizes & $\sigma_{x}$ & $0.42 \mathrm{~mm}$ \\
& $\sigma_{y}$ & $0.06 \mathrm{~mm}$ \\
Bunch length & $\sigma_{z}$ & $4 \mathrm{~mm}$ \\
Synchrotron tune & $\nu_{s}$ & 0.024 \\
Betatron tune & $\nu_{x(y)}$ & $45.51 / 43.57$ \\
Damping time & $\tau_{x(y)} / T_{0}$ & $4000 \mathrm{turn}$ \\
Chamber radius & $R$ & $0.05 \mathrm{~m}$ \\
\hline \hline
\end{tabular}

their arrival time $\left[t=(k-n / M) T_{0}\right]$,

$$
\begin{aligned}
Y_{m}(t)= & a_{m} \sum_{k=1}^{\infty} \sum_{n=1}^{M} \exp \left[-i \omega_{\beta} t+2 \pi i m n / M\right] \\
& \times \delta\left[t-(k-n / M) T_{0}\right] \\
= & a_{m} \sum_{k=1}^{\infty} \sum_{n=1}^{M} \exp \left[-i\left(\omega_{\beta}+m \omega_{0}\right) t\right] \\
& \times \delta\left[t-(k-n / M) T_{0}\right]
\end{aligned}
$$

where $T_{0}$ is the revolution time and $\omega_{0}=2 \pi / T_{0}$. The index $k$ denotes the revolution number, and $n$ denotes the bunch number. The phase, $\left(\omega_{\beta}+m \omega_{0}\right) t=\left(\nu_{\beta}+m\right) \omega_{0} t$, as a function of time, represents the betatron phase variation of each bunch during the passage of the bunch train at the position monitor, where $\nu_{\beta}=\omega_{\beta} / \omega_{0}$ is the betatron tune. For $0<\nu_{\beta}+m<M / 2$ or $M / 2<\nu_{\beta}+m<M$ (equivalently $-M / 2<\nu_{\beta}+m<0$ ), the betatron phase advances or retards as time goes by, respectively. We call the advanced and retarded modes those for which $0<$ $\nu_{\beta}+m<M / 2$ and $M / 2<\nu_{\beta}+m<M$, respectively, in this paper.

The Fourier transformation of the monitor output $\left(Y_{m}\right)$ is expressed as

$$
F_{m}(\omega)=a_{m} M \omega_{0} \sum_{p=-\infty}^{\infty} \delta\left(\omega-\omega_{\beta}-m \omega_{0}-p M \omega_{0}\right) .
$$

Unstable modes are identified by peaks of betatron sideband frequency $\left(\omega_{\beta}+m \omega_{0}+p M \omega_{0}\right)$ in the Fourier spectrum.

The electron cloud experiences the bunch position $Y_{m}$ at its location $s_{e}$. Since the motions of electrons at different locations do not have any correlation, the instability is insensitive to location and to the number of locations, unless the tune shift due to the cloud is large. As is known in the case of ordinary wakes, the integrated wake force determines the characteristics of the instability.
The characteristics of the instability are determined by the collective motions of the electrons. The electron motions induced by the passage of a bunch perturb the following bunches. For example, if electrons oscillate coherently with a frequency $(\omega)$, a mode $m$, determined by $\omega \approx$ $\pm\left(\omega_{\beta}+m \omega_{0}\right)+p M \omega_{0}$, is induced. An ordinary wake force has a defocusing nature just after the occurrence of a perturbation, i.e., a positive shift of a bunch induces a positive kick in the nearby following bunches. An unstable mode with the frequency $\omega \approx(p M-m) \omega_{0}-\omega_{\beta}$ is induced by the defocusing wake force. That is, a retarded mode is induced at a frequency of $\omega<M \omega_{0} / 2$. Note that the frequency of an electron cloud which causes coupledbunch instability satisfies $\omega<M \omega_{0} / 2$. In the opposite case, where the wake force has a focusing nature, an advanced mode is induced.

So far we have discussed modes of instability for bunches with equal spacing. In fact, bunches are often not filled uniformly. If anything, it is more common to fill bunches with a gap, unequal bunch population or irregular bunch spacing. The Fourier transformation of the signal is expressed as

$$
F_{m}=a_{m} \omega_{0} \sum_{q=-\infty}^{\infty} \delta\left(\omega-\omega_{\beta}-m \omega_{0}-q \omega_{0}\right) f(\boldsymbol{b}, q) .
$$

$f(\boldsymbol{b}, q)$ characterizes the contamination of the mode spectrum due to the irregular bunch filling,

$$
f(\boldsymbol{b}, q)=\sum_{n=1}^{M} b_{n} \exp (-2 \pi i n q / M)
$$

where $b_{n}$ is the bunch population of the $n$th bunch. The term including the summation is zero except for $q=p M$, due to cancellation of the phase angle, if $b_{n}$ is a constant. Even if $b_{n}$ is modulated with a small amplitude, or $b_{n}=0$ for some $n$, the term with $q=p M$ is dominant as long as the cancellation is effective. For example, bunches up to the 1209th bucket are filled for $M=1280$ in typical operation at KEKB: $b_{n}=N_{p}$ and $b_{n}=0$ for $n \leq 1209$ and $n>1209$, respectively. Note that the harmonic number is $H=5120$ and bunches are located every 4 buckets, $M=$ $H / 4=1280$. The contamination is evaluated as $\mid f(\boldsymbol{b}, q \neq$ $p M) / f(\boldsymbol{b}, p M) \mid<0.06$ for $|q-p M|<10$ at the most. In addition, the coupled-bunch instability caused by an electron cloud has a large frequency spread. Therefore the spectrum is not affected by the detailed structure of the bunch fill pattern.

\section{EQUATION OF MOTION}

We will now focus on the dipole mode of the coupledbunch instability. A positron bunch can be characterized by its transverse and longitudinal position (dipole moment) as a function of $s$, ignoring the internal structure of the bunch. Interactions between bunches and electrons in a cloud are determined by the transverse and longitudinal profiles of 
the bunches. The profiles are assumed to be Gaussian with standard deviation determined by the emittance and the average beta function in the transverse and longitudinal directions. The motion of each bunch is determined by the transformation representing lattice magnets and the interactions with electrons, while the motions of the electrons are determined by the interactions with the bunches, space charge forces between the electrons, and any magnetic field. The equations of motion are written as

$$
\begin{gathered}
\frac{d^{2} \boldsymbol{x}_{p}}{d s^{2}}+K(s) \boldsymbol{x}_{p}=\frac{r_{e}}{\gamma} \sum_{e=1}^{N_{e}} \boldsymbol{F}\left(\boldsymbol{x}_{p}-\boldsymbol{x}_{e}\right) \delta_{P}\left(s-s_{e}\right), \\
\frac{d^{2} \boldsymbol{x}_{e}}{d t^{2}}=2 r_{e} c^{2} \sum_{p=1}^{N_{b}} \boldsymbol{F}\left(\boldsymbol{x}_{e}-\boldsymbol{x}_{p}\right) \delta_{P}\left[t-t_{p}\left(s_{e}\right)\right] \\
+\frac{e}{m_{e}} \frac{d \boldsymbol{x}_{e}}{d t} \times \boldsymbol{B}-2 r_{e} c^{2} \frac{\partial \boldsymbol{\phi}}{\partial \boldsymbol{x}}
\end{gathered}
$$

where subscripts $p$ and $e$ of $\boldsymbol{x}$ denote positron and electron, respectively, $r_{e}$ is the classical electron radius, $m_{e}$ is the electron mass, $c$ is the speed of light, $e$ is the electron charge, $\phi$ is the electric potential due to electrons, $\delta_{P}$ is the periodic delta function for the circumference, and $F$ is the Coulomb force in two-dimensional space given by the Bassetti-Erskine formula [11]. In calculating the interaction, a single kick is too strong to simulate electron motion, especially for electrons near the beam, therefore, in actual calculation, we replace $\delta_{P}\left(s-s_{e}\right)$ in Eqs. (6) and (7) by a broadened function of width $\sigma_{z}$, and $\boldsymbol{F}$ is the sum of several (typically 10) kicks (or slices) using the formula. Each slice in a bunch has a unique transverse coordinate, since only the dipole moment of the bunch is considered.

The normalized electric potential, which represents interactions between electrons, is determined by the Poisson equation,

$$
\triangle_{\perp} \phi(x)=\sum_{a=1}^{N_{e}} \delta\left(x-x_{e, a}\right) .
$$

The space charge force is solved with a Green function including the circular (cylindrical) boundary condition, since the vacuum chamber is a cylindrical pipe at KEKB.

The electron cloud buildup is estimated by using Eq. (7) alone. Initial conditions for the electrons are given by the model shown in Fig. 1 [2]. We consider the longitudinal position of the ring characterized by $s_{e}$. Electrons are created when the positron bunch arrives at $s_{e}$, at time $t=$ $t\left(s_{e}\right)$. Photoemission due to synchrotron radiation is the dominant electron-production process for positron rings. The ring-averaged number of photons per meter hitting the chamber wall is given by

$$
n_{\gamma}\left(/ m \cdot e^{+}\right)=\frac{5 \pi}{\sqrt{3}} \frac{\alpha \gamma}{L},
$$

where $\alpha$ and $\gamma$ are $1 / 137$ and the relativistic factor, re-

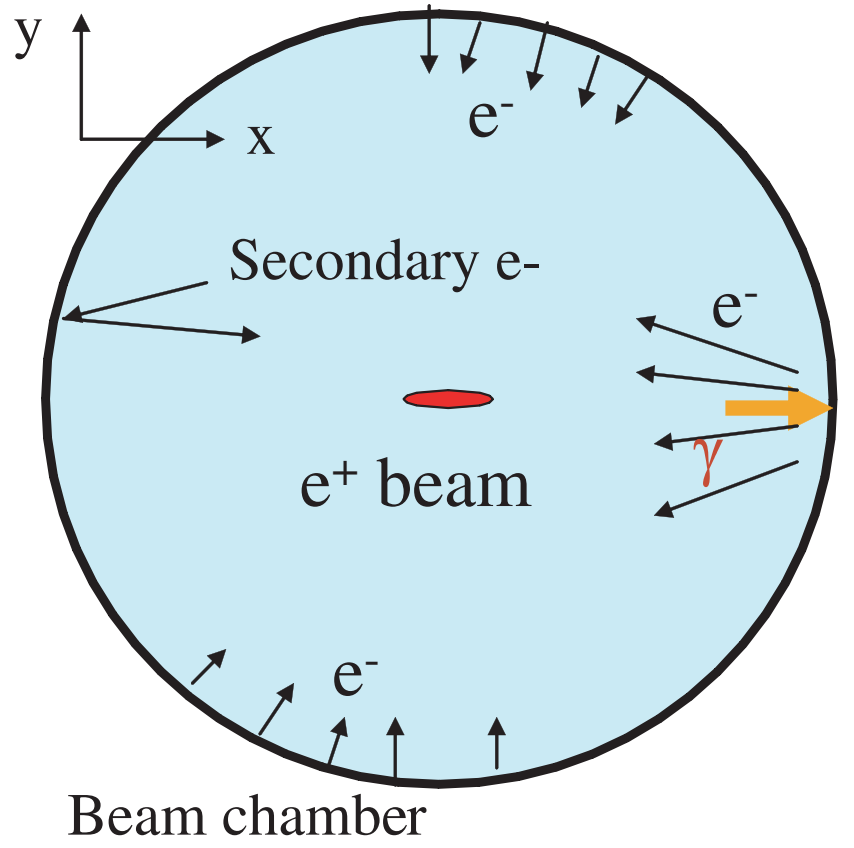

FIG. 1. (Color) Schematic view of simulation of electron cloud buildup. A large part of electrons is produced at a position illuminated by synchrotron radiation, while considerable part are also produced uniformly on the chamber surface due to reflected photons.

spectively. The number of photoelectrons produced by a positron at the chamber is given by

$$
n_{e \gamma}\left(/ m \cdot e^{+}\right)=n_{\gamma} Y_{\gamma}
$$

The direct photoemission rate was estimated to be $Y_{\gamma} \sim$ 0.1 . The typical production rate is $n_{e \gamma}=0.015 e^{-} /(\mathrm{m}$. $\left.e^{+}\right)$at KEKB. For a proton ring, proton loss is considered to be the dominant source. The rate is $n_{\text {ploss }}=10^{-6} \sim$ $10^{-5} e^{-} /(m \cdot p)$. Ionization is a common source to both positron and proton rings. The electron production is generally very small at the vacuum pressure of an ordinary accelerator: $\quad n_{I} \sim 1 \times 10^{-8} e^{-} /\left[m \cdot e^{+}(p)\right]$ at $2 \times$ $10^{-7} \mathrm{~Pa}$.

A secondary electron is produced with a probability $\left[\delta_{2}(E)\right]$ when an electron is absorbed at the chamber wall. The probability, which is a function of energy of the incident electron $(E)$, is given typically as follows [12-14]:

$$
\begin{aligned}
\delta_{2}(E)= & \delta_{0} \exp \left(-5 E / E_{\max }\right)+\delta_{2, \max } \frac{E}{E_{\max }} \\
& \times \frac{1.44}{0.44+\left(E / E_{\max }\right)^{1.44}} .
\end{aligned}
$$

The electrons build up to a certain density which is determined by the balance of creation and absorption of electrons moving under the influence of forces, and by the initial conditions. 
Photons emitted by the synchrotron radiation from a bending magnet hit a narrow strip region on the side of the chamber wall as shown in Fig. 1. The majority of photoelectrons are produced in the strip region illuminated by the synchrotron radiation, especially near the downstream edge of the bending magnet. Since electrons are attracted to the beam, they may be distributed with planar symmetry along the $x$ axis. A considerable number of photons are reflected and hit the wall again. The reflected photons are not considered to be localized in a narrow region. We therefore assume some electrons are produced uniformly on the vacuum chamber wall. The electrons produced uniformly could dominate far downstream of the bending magnet, where they would be distributed with azimuthal symmetry.

The secondary emission rate has been measured to be $\delta_{2, \max }=1.4$ for a pure copper surface, while it is reduced to 1.2 or less by conditioning due to the beam $[15,16]$. We use the secondary rates, $\delta_{2, \max }=1.0$ or 1.5 at $E_{\max }=$ $200 \mathrm{eV}$, in simulations. When secondary emission dominates, the distribution approaches azimuthal symmetry for primary electrons for both the strip region and the uniform emission region.

The electron distribution strongly depends on the magnetic field. In a weak solenoid $\left(B_{z} \sim 10 \mathrm{G}\right)$, electrons are distributed in a shell with a width of the cyclotron radius near the chamber surface. In a strong bending magnet $B_{y} \sim$ $1 \mathrm{~T}$, electrons are distributed with planar symmetry along the $y$ axis. Figure 2 shows four typical distributions of electron clouds. Plots (a) and (b) depict electron distribution in a drift space. In plot (a), electrons are produced at an illuminated position in the drift space and an additional $30 \%$ of them are produced uniformly, with the secondary efficiency set to $\delta_{2, \max }=1.0$. In plot (b), electrons are produced uniformly in the drift space and the secondary emission efficiency is set to $\delta_{2, \max }=1.0$. Plots (c) and (d) depict electron distributions in a weak solenoid $\left(B_{z}=\right.$
$30 \mathrm{G})$ and a strong bending magnet $\left(B_{y}=\infty\right)$, respectively. Electrons are produced uniformly in both plots. Electrons produced at the illuminated position have a similar distribution in the weak solenoid magnet, except a considerable number of electrons are localized near the illuminated position. In the strong bending magnet, electrons produced at the illuminated position are completely localized only at that position. $B_{y}=\infty$ can be assumed in the bending magnet, since the cyclotron radius for $B \sim 1 \mathrm{~T}$ is smaller than the beam size.

The electron distribution is reflected in the characteristics of the coupled-bunch instability; the coupled-bunch instability is caused by the interaction of the beam with a collectively moving electron cloud, and the motions of the cloud electrons are closely related to the cloud distribution. The collective motion and the distribution of the electron cloud depends on the location in the ring: i.e., near or far downstream of a bending magnet, in drift space, in bending magnet, in solenoid magnet or in other conditions. Characteristics of the coupled-bunch instability are determined by the averaged and/or integrated collective motion of electron clouds in the whole ring. For the evaluation of the instability, we assume that the entire length of the ring is a drift space, or a solenoid, or a strong dipole field, and we consider either of the electron-production conditions. The true instability growth rate and mode spectrum for an actual ring could be computed by an appropriate weighted average of the these separate results.

\section{WAKE FIELD AND GROWTH RATE OF THE COUPLED-BUNCH INSTABILITY}

The coupled-bunch instability is simulated by solving Eqs. (6)-(8) with initial conditions self-consistently. It is not very difficult to simulate the evolution of each bunch amplitude by observing its instability growth. However this method requires great computer resources and is time consuming. In addition, such a method does not often
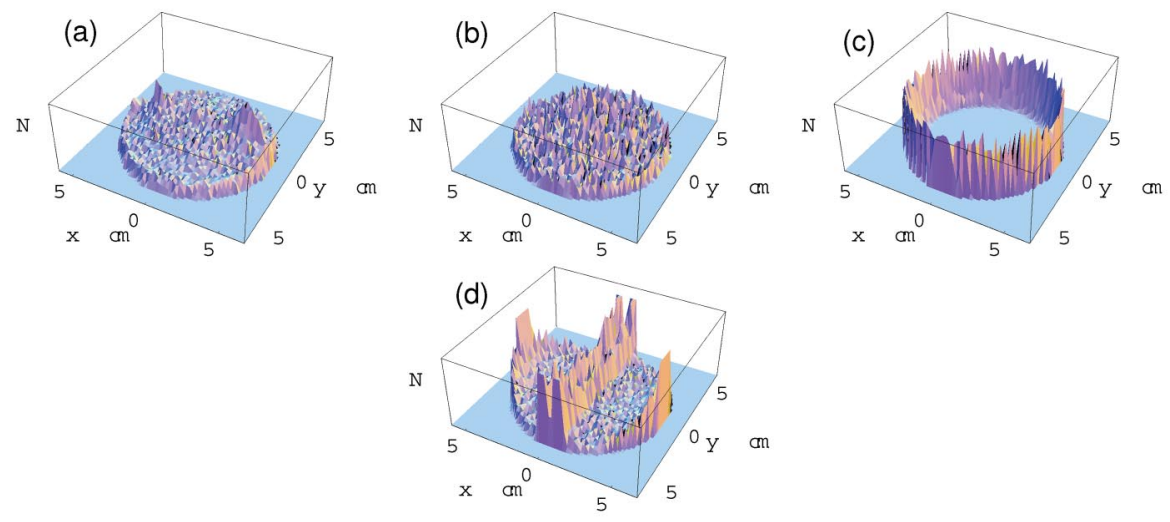

FIG. 2. (Color) Typical electron cloud distribution in the transverse $x-y$ plane. The number of electrons in an arbitrary unit is plotted in the vertical axis. Plots (a) and (b) depict electron distributions in a drift space, where (a) and (b) are for electrons produced mainly at the illuminated position, and produced uniformly, respectively. Plots (c) and (d) depict electron distributions in weak solenoid and strong bending magnets, respectively. 
give a physical picture of the instability. An approximation can make the physical picture transparent. We discuss a (semi)analytic method and direct self-consistent solution of Eqs. (6)-(8) in this and following sections. The mechanism and causes of the instability are similar to an ordinary instability due to the vacuum chamber structure. The correlation between bunches is induced by the coherent motion of electrons in the cloud or the electromagnetic field in the chamber.

Ordinary instability theory is based on wake fields. The vertical force which a bunch at $z$ experiences is expressed by the convolution of the wake field $\left(W_{1, y}\right)$ and the dipole moments of the bunches ahead of the bunch $\left(z_{i}<z_{j}\right)$,

$$
F_{y}\left(z_{i}\right)=\frac{N_{p} r_{e}}{\gamma} \sum_{j>i}^{\infty} W_{1, y}\left(z_{i}-z_{j}\right) y_{j}\left(z_{j}\right)
$$

where the wake field $W_{1, y}(z)$ is a function of the distance between the analyzing bunch $\left(z_{i}\right)$ and the bunches with a dipole moment $\left(z_{j}\right)$. Bunches with larger values of $i$ are in front of those with smaller values. The convolution is based on the linearity and superposition of the wake force.

The force [Eq. (12)] for a bunch train uniformly filled with bunch spacing $L_{\mathrm{sp}}$ induces a coupled-bunch instability characterized by a dispersion relation,

$$
\begin{aligned}
\left(\Omega_{m}-\omega_{\beta}\right) L / c= & \frac{N_{p} r_{e} c}{2 \gamma \omega_{\beta}} \sum_{\ell=1}^{N_{w}} W_{1}\left(-\ell L_{\mathrm{sp}}\right) \\
& \times \exp \left(2 \pi i \ell \frac{m+\nu_{\beta}}{M}\right),
\end{aligned}
$$

where $N_{w}$ is the range of the wake field. The imaginary part of $\Omega_{m} L / c=T_{0} / \tau_{m}$ is the growth rate per revolution for the $m$ th mode. Bunches oscillate with a mode characterized by $m$ as

$$
y_{m}\left(z_{j}\right)=a_{m} \exp \left[-i \Omega_{m} t+2 \pi i m j / M\right],
$$

where $M$ is the number of bunches, assuming equal spacing. The real part of $\Omega_{m}$ is close to $\omega_{\beta}$.

We introduce the wake field for the beam-electron cloud interaction. The wake field is related to the collective motion of the electrons. Electrons near the beam center oscillate with the frequency

$$
\omega_{e, x(y)}=\sqrt{\frac{2 \lambda_{p} r_{e} c^{2}}{\sigma_{x(y)}\left(\sigma_{x}+\sigma_{y}\right)}},
$$

where $\sigma_{x(y)}$ is the horizontal (vertical) beam size. The bunches arrive frequently but the spacing between bunches is much longer than the bunch length for the positron ring, $\omega_{e} L_{\mathrm{sp}} / c \gg 1$, therefore electrons are not trapped by the beam potential, with the result that most of the electrons move in a nonlinear region. In this situation, it is not clear whether the wake force possesses linearity and superposition.
We are interested in coherent effects for a small perturbation of the dipole amplitude of the beam. Though the motion of each electron is nonlinear, an averaged force may have linearity and superposition characteristics. For example, an electric force in a cylindrical electron distribution is linear, though it has a $1 / r^{2}$ dependence for each electron.

From a macroscopic point of view, there may be some characteristic frequency, for example, the cyclotron frequency $\left(\omega_{c}=e B / m_{e}\right)$ for a finite magnetic field. If the cloud is approximated by a static Gaussian distribution with the horizontal and vertical rms sizes, $\Sigma_{x}$ and $\Sigma_{y}$, larger than those of beam, the characteristic frequency is now expressed by

$$
\omega_{G, y}=\sqrt{\frac{2 \bar{\lambda}_{p} r_{e} c^{2}}{\Sigma_{y}\left(\Sigma_{x}+\Sigma_{y}\right)}},
$$

where $\bar{\lambda}_{p}=N_{p} / L_{\mathrm{sp}}$ is an average beam line density.

An electron experiences a strongly nonlinear force from the beam. Coherent motion of the electron cloud damps rapidly due to Landau damping, with the result that the wake field has a broadband component and the mode spectrum is widely spread. In other words, the electrons are not trapped and are absorbed at the chamber, therefore the memory of the dipole moment of a bunch is soon lost from the cloud. If a magnetic field is applied, Landau damping may be suppressed by cyclotron or related motions. For example, in a solenoid field, electrons move in the transverse plane at the cyclotron frequency. The frequency of the motion does not spread very much; the cyclotron frequency depends only on the magnetic field strength. The electrons have a long life time in the chamber. The magnetic field works to reduce electron density near the beam and weakens the strength of the wake field, while the increase in the quality factor of the wake field worsens the instability.

\section{A. Analytic method: broadband resonator model}

Some trials using an analytic method have been done to study the electron cloud induced coupled-bunch instability $[17,18]$. The difficulty with analytic theory for the coupledbunch instability is due to the fact that individual electrons in the cloud do not experience linear harmonic motion at all. Therefore we have treated the collective motion of the whole cloud and assumed the linearity of the interaction with the beam.

We discuss the interaction of the beam with a rigid Gaussian cloud with average sizes $\Sigma_{x}$ and $\Sigma_{y}$ here. In this model, the linearity and superposition conditions are satisfied automatically for small amplitudes, $x, y \ll$ $\Sigma_{x}, \Sigma_{y}$. We expect in this model that the cloud moves with the frequency $\omega_{G, y}$ overall, though individual electrons move at various frequencies. This approach is similar to that used for the electron cloud induced single-bunch 
instability. For the single-bunch instability, the local electron distribution is important, so the electron frequency in a bunch [Eq. (15)] is the characteristic frequency [19].

We use the cloud size $\Sigma_{x} \times \Sigma_{y}=4 \times 1.5 \mathrm{~cm}^{2}$, as is estimated in Fig. 2(a). The characteristic frequency is $\omega_{G, y}=2 \pi \times 18 \mathrm{MHz}$, and the phase advance across the bunch spacing ( $8 \mathrm{~ns}$ ) is less than $1, \omega_{G, y} L_{\mathrm{sp}} / c=0.9$ for $L_{\mathrm{sp}}=2.4 \mathrm{~m}$, therefore the coasting beam approximation can be applied. For larger bunch spacing, the cloud cannot exist stably. The equation of motion is expressed as

$$
\begin{aligned}
\frac{d^{2} y_{p}(s, z)}{d s^{2}}+\left(\frac{\omega_{\beta, y}}{c}\right)^{2} y_{p}(s, z)= & -\left(\frac{\omega_{p, y}}{c}\right)^{2}\left\{y_{p}(s, z)\right. \\
& \left.-y_{e}[s,(s+z) / c]\right\},
\end{aligned}
$$

$$
\frac{d^{2} y_{e}(s, t)}{d t^{2}}+2 \alpha \frac{d y_{e}(s, t)}{d t}=-\omega_{G, y}^{2}\left[y_{e}(s, t)-y_{p}(s, c t-s)\right],
$$

where $\omega_{\beta, y}$ denotes the angular betatron frequency free from electron interaction. The two coefficients $\omega_{G, y}$ and $\omega_{p, y}$ characterize the linearized force between beam and cloud, and are given by Eq. (16) and

$$
\omega_{p, y}^{2}=\frac{2 \lambda_{e} r_{e} c^{2}}{\gamma\left(\Sigma_{x}+\Sigma_{y}\right) \Sigma_{y}},
$$

where $\lambda_{e}$ and $\bar{\lambda}_{p}$ are the average line densities of the cloud and the beam. $\alpha=\omega_{G, y} / 2 Q$, which depends on the quality factor $(Q)$, characterizes the damping of the collective motion of the electron cloud due to the frequency spread of each electron.

The two equations are combined using the wake field

$$
\frac{d^{2} y_{p}(s, z)}{d s^{2}}+\left(\frac{\tilde{\omega}_{\beta, y}}{c}\right)^{2} y_{p}(s, z)=\frac{\bar{\lambda}_{p} r_{e}}{\gamma} \int_{z}^{\infty} W_{1}\left(z-z^{\prime}\right) y_{p}\left(s, z^{\prime}\right),
$$

where $\tilde{\omega}_{\beta, y}^{2}=\omega_{\beta, y}^{2}+\omega_{p, y}^{2} . W_{1}$, which is the wake field for the beam-cloud interaction, is expressed as

$$
W_{1}(z)\left[\mathrm{m}^{-2}\right]=c \frac{R_{S}}{Q} \frac{\omega_{G, y}}{\hat{\omega}} \exp \left(\frac{\alpha}{c} z\right) \sin \left(\frac{\hat{\omega}}{c} z\right)
$$

for $z<0$, where

$$
c R_{S} / Q=\frac{\lambda_{e}}{\bar{\lambda}_{p}} \frac{2 L}{\left(\Sigma_{x}+\Sigma_{y}\right) \Sigma_{y}} \frac{\omega_{G, y}}{c},
$$

and $\hat{\omega}^{2}=\omega_{G, y}^{2}-\alpha^{2}$.

The frequencies of individual electrons are spread over a very wide range, as previously discussed. For now we will take $Q=1$ - that is, the wake field induced by the electron cloud is considered as a broadband resonator with $Q=1$.
The coupled-bunch instability is estimated by the dispersion relation Eq. (13) for the broad band wake field. $N_{w}$ is taken to be $10 \sim 20$ at most for the low $Q$. Figure 3 shows the wake field and the growth rate of each coupledbunch mode given by Eq. (13). $L_{\mathrm{sp}}=2.4 \mathrm{~m}(8 \mathrm{~ns})$ and $\bar{\lambda}_{p}=N_{p} / L_{\mathrm{sp}}=2.1 \times 10^{10} \mathrm{~m}^{-1}$ are used in the estimation. The cloud density is determined by the buildup simulation as $\lambda_{e}=1.5 \times 10^{10} \mathrm{~m}^{-1}$. The size is $\Sigma_{x} \times$ $\Sigma_{y}=4 \times 1.5 \mathrm{~cm}^{2} . \quad c R_{S} / Q=2.0 \times 10^{6} \mathrm{~m}^{-2}, \quad \hat{\omega} / c=$ $0.33 \mathrm{~m}^{-1}$, and $\alpha / c=0.19 \mathrm{~m}^{-1}$ are given by the parameters. The wake field has a somewhat longer range than that given by the semianalytical method, as shown later in Fig. 5. The amplitude given by this model is consistent with that given by the semianalytical method. The growth time obtained is around 4-5 turns, which is twice as fast or more than that given by semianalytical and tracking methods. The wake field range and growth time can agree with the semianalytical result by taking a smaller cloud $\Sigma_{x(y)} \sim$ $0.7 \mathrm{~cm}$.

\section{B. Semianalytical method}

We now discuss the semianalytic method, in which the wake force is calculated numerically and the growth rate of each coupled-bunch mode is then evaluated by Eq. (13) [2]. This method was used to understand the coupled-bunch instability observed at the KEK Photon Factory [1].

We imagine bunches interacting with the cloud at a position $s$. The momentum kick experienced by the $i$ th bunch is expressed as

$$
\Delta y_{p, i}^{\prime}=\frac{N_{p} r_{e}}{\gamma} \sum_{j>i}^{i+N_{w}} W_{1}\left(z_{i}-z_{j}\right) y_{p, j},
$$

where $y^{\prime}=d y / d s$. This equation is generally used to
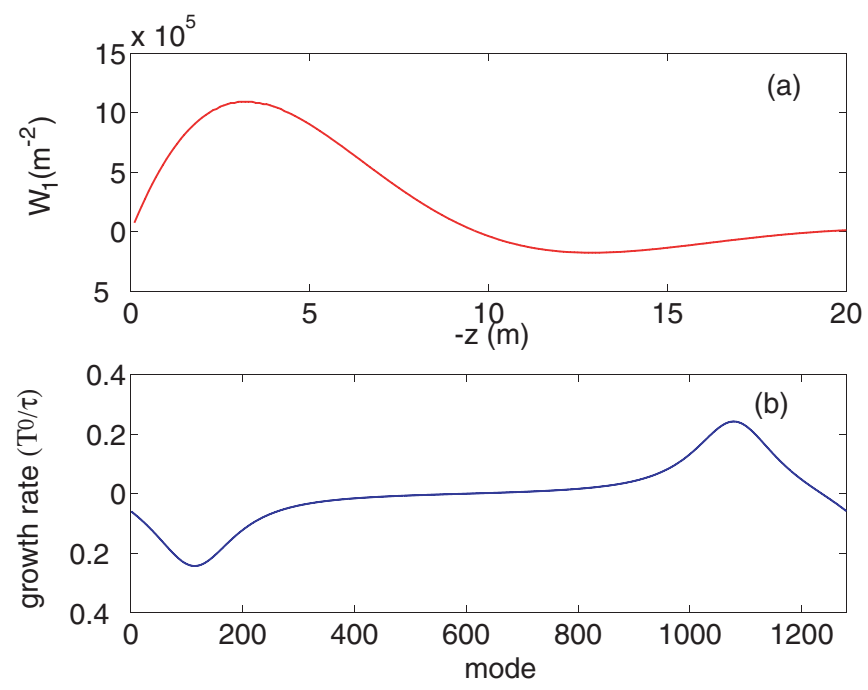

FIG. 3. (Color) Wake field (a) and growth rate $\left[T_{0} / \tau\left(\omega_{m}\right)\right]$ of each coupled-bunch mode (b) given by the analytic method at KEKB (8 ns spacing). 
simulate beam motion for a given wake field $W_{1}$. We use this formula to evaluate the wake field induced by the electron cloud. Equation (23) is a linear matrix equation connecting $\Delta y_{p, i}^{\prime}$ to $y_{p, j}$. The matrix element $W_{1}\left(z_{i}-z_{j}\right)$, is determined from the momentum kick on a bunch $\Delta y_{p, i}^{\prime}$ due to the displacement of another bunch $y_{p, j}$. The momentum kick $\left(\Delta y_{p, i}^{\prime}\right)$ is calculated by the simulation when the displaced bunch at $y_{p, j}$ passes through an electron cloud. The displaced bunch is, of course, ahead of the kicked bunch.

The wake field is evaluated by computer simulation as follows:

(i) Primary electrons are created at every bunch passage on the chamber surface with the line density $n_{e \gamma}$ and are accelerated by the beam force. Secondary electrons are created upon absorption of an electron with an energy $\left(E_{\mathrm{abs}}\right)$ at the rate $\delta_{2}\left(E_{\mathrm{abs}}\right)$.

(ii) The creation process is repeated until the cloud density saturates at a certain value. These two steps are the same in the buildup simulation.

(iii) A bunch with a slight displacement $\left(y_{0}\right)$ passes through the cloud, and then trailing bunches without displacement pass through the chamber center.

(iv) The creation process is repeated for the displaced and following bunches.

(v) The trailing bunches experience forces from the cloud, because the cloud is perturbed by the passage of the displaced bunch. The wake field is calculated from these forces.

Linearity and superposition are assumed in Eq. (23); the kick $\Delta y_{p, i}^{\prime}$ is the result of a sum of bunch displacements, $y_{p, j}$. The simulation needs to check whether this is correct or not- the wake force induced by a bunch for several amplitudes of displacement, and that induced by two or more bunches should be compared.

The growth rate of each bunch oscillation mode is given by the dispersion relation, Eq. (13). The dispersion relation is based on the properties of linearity an superposition for the displacement $y_{p, j}$ in Eq. (23). Exponential growth, determined by the imaginary part of $\Omega_{m}$, is found after satisfying linearity and superposition.

Figure 4 shows the wake force given by this method, where the bunch spacing is $8 \mathrm{~ns}$. Primary electrons are produced at a photon-illuminated position, an additional $30 \%$ of them are produced uniformly on the chamber surface, and secondary electrons are produced at the rate, $\delta_{2 \max }=1.0$. To check the linearity, the wake force $\left(W_{1, y} y_{0}\right)$ for $y_{0}=1,2,5 \mathrm{~mm}$ is evaluated. Here the 200th bunch is displaced and the forces which the following bunches experience from the electron cloud are plotted. The wake force has a peak at the 201st bunch, which is linear with the amplitude of initial displacement $\left(y_{0}\right)$. It oscillates 1 or 2 periods with a much smaller amplitude than at the peak. Linearity of the wake force is satisfied up

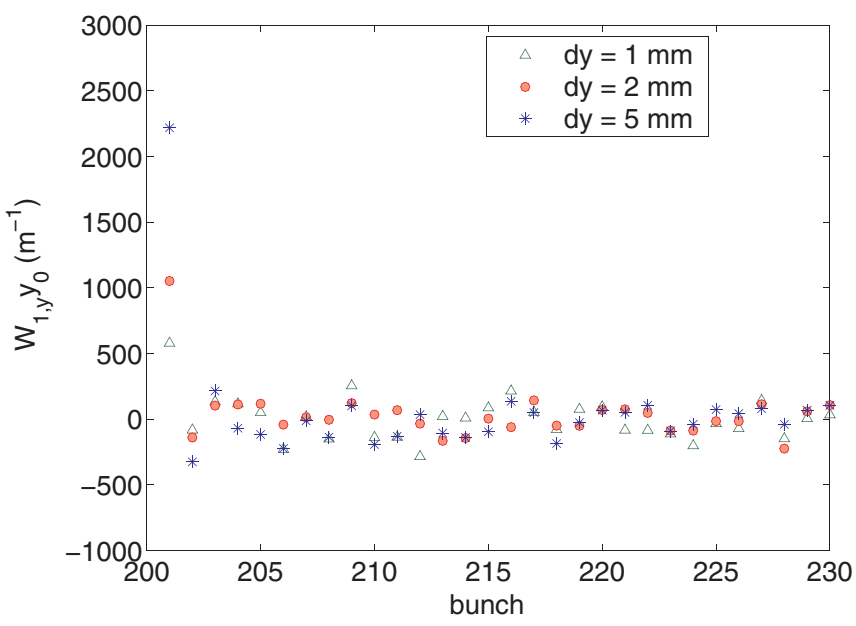

FIG. 4. (Color) Vertical wake force and cloud line density along the bunch train at KEKB. Bunch repetition is $8 \mathrm{~ns}$. Three types of points are given for the displacement, $y_{0}=1,2$, and $5 \mathrm{~mm}$. Primary electrons are produced at a photon-illuminated position, $30 \%$ of them are produced uniformly on the chamber surface, and secondary electrons are produced with the rate, $\delta_{2 \max }=1.0$.

to no more than the 210th bunch. The direction of the wake force is the same as that of the displacement - that is, it is a "defocusing" force for a bunch train. This characteristic is the same as that of an ordinary wake force.

The wake force after the 210th bunch is considered to be numerical noise in the simulation or to be an effect of nonlinear motion of electrons. Electron cloud average line density reaches a saturation level of $\sim 1.5 \times$ $10^{10} \mathrm{~m}^{-1}$ after $\sim 100$ bunch passages. The beam line density is $5 \times 10^{10} / 2.4 \sim 2 \times 10^{10} \mathrm{~m}^{-1}$, which is $75 \%$ of the

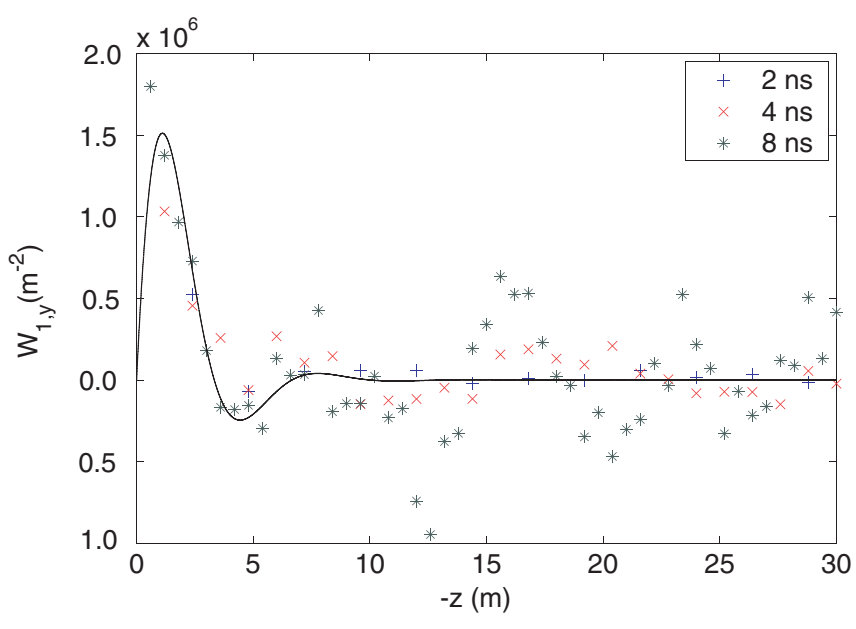

FIG. 5. (Color) Vertical wake field for 2, 4, and $8 \mathrm{~ns}$ spacing as a function of $z$ at KEKB, where $y_{0}=2 \mathrm{~mm}$. Primary electrons are produced at a photon-illuminated position, $30 \%$ of them are produced uniformly on the chamber surface, and secondary electrons are produced with the rate, $\delta_{2 \max }=1.0$. The solid line correspond to the wake field with the broadband resonator model, $\Sigma_{x}=\Sigma_{y}=0.7 \mathrm{~cm}^{2}$ and $\lambda_{e}=0.1 \times 10^{10} \mathrm{~m}^{-1}$. 
neutralization level. The same simulation was performed for the higher secondary efficiency $\delta_{2 \max }=1.5$. The cloud line density and strength of the wake force increased 2 times, but did not change the shape of the wake force.

Figure 5 shows the wake field for various bunch spacings, keeping the total current constant: i.e., the bunch populations for 2 and $4 \mathrm{~ns}$ spacings are $N_{p} / 4$ and $N_{p} / 2$, respectively. Conditions for electron production are the same as previously. The wake field does not strongly depend on the spacing as far as can be seen from strength and range. In the figure, the wake field, which is given by the broadband resonator model for $\Sigma_{x}=\Sigma_{y}=0.7 \mathrm{~cm}^{2}$ and $\lambda_{e}=0.1 \times 10^{10} \mathrm{~m}^{-1}$, is plotted, where $c R_{S} / Q=$ $3.2 \times 10^{6} \mathrm{~m}^{-2}, \hat{\omega} / c=0.94 \mathrm{~m}^{-1}$, and $\alpha / c=0.54 \mathrm{~m}^{-1}$. It agrees well with that of the semianalytic method. This fact indicates how many electrons contribute to the coupled-bunch instability.

The wake force does not depend much on the emittance, and can therefore occur in the horizontal direction. Figure 6 shows the horizontal wake force for $x_{0}=$ $5 \mathrm{~mm}$. Here the wake force is calculated for $8 \mathrm{~ns}$ spacing. Primary electrons are produced at an illuminated position of photon and $30 \%$ of them are produced uniformly on the chamber surface. Two plots, (a) and (b), are given for two secondary rates, $\delta_{2, \max }=1.0$ and 1.5 , respectively. For the lower secondary rate, the electron distribution is flat along the $x$ plane with a thickness of $1-2 \mathrm{~cm}$. The direction of the wake force is opposite to that of the displacement; that is, it is a "focusing" wake for the bunch train. For the higher secondary rate, the electron cloud is distributed with cy-
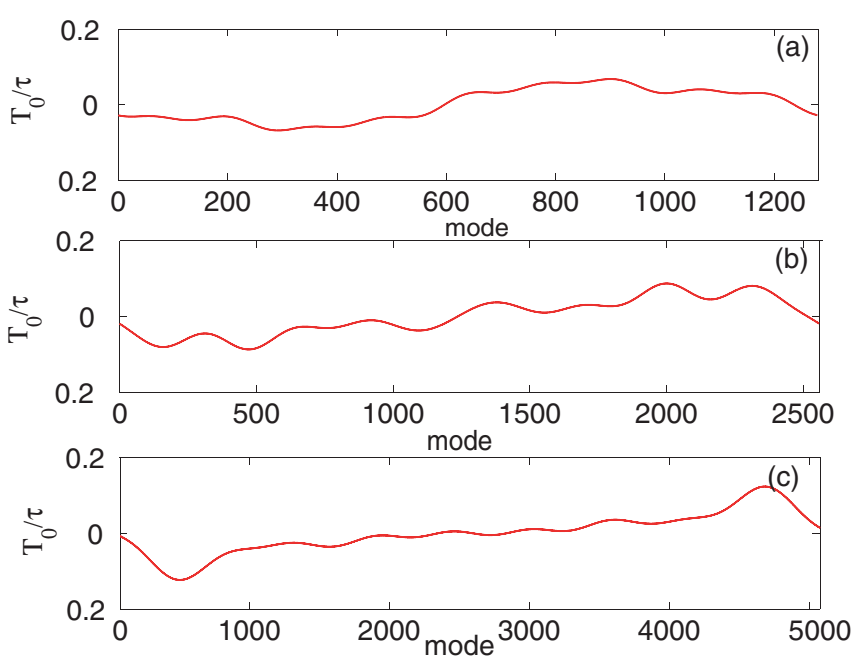

FIG. 7. (Color) Growth rate of vertical coupled-bunch instability estimated by the wake field in Fig. 5. A positive rate indicates instability. Plots (a), (b), and (c) depict mode spectra for 2, 4, and $8 \mathrm{~ns}$ spacing. Primary electrons are produced at a photonilluminated position, $30 \%$ of them are produced uniformly on the chamber surface, and secondary electrons are produced with the rate, $\delta_{2 \max }=1.0$.

lindrical symmetry, with the result that the wake force is similar to the vertical one. The same simulations was performed for electrons produced uniformly at the lower and higher secondary rates. The wake force has a shape like in plot (b) as is expected for both cases.

Unstable modes of the coupled-bunch instability and their growth rates are calculated by Eq. (13). Figure 7
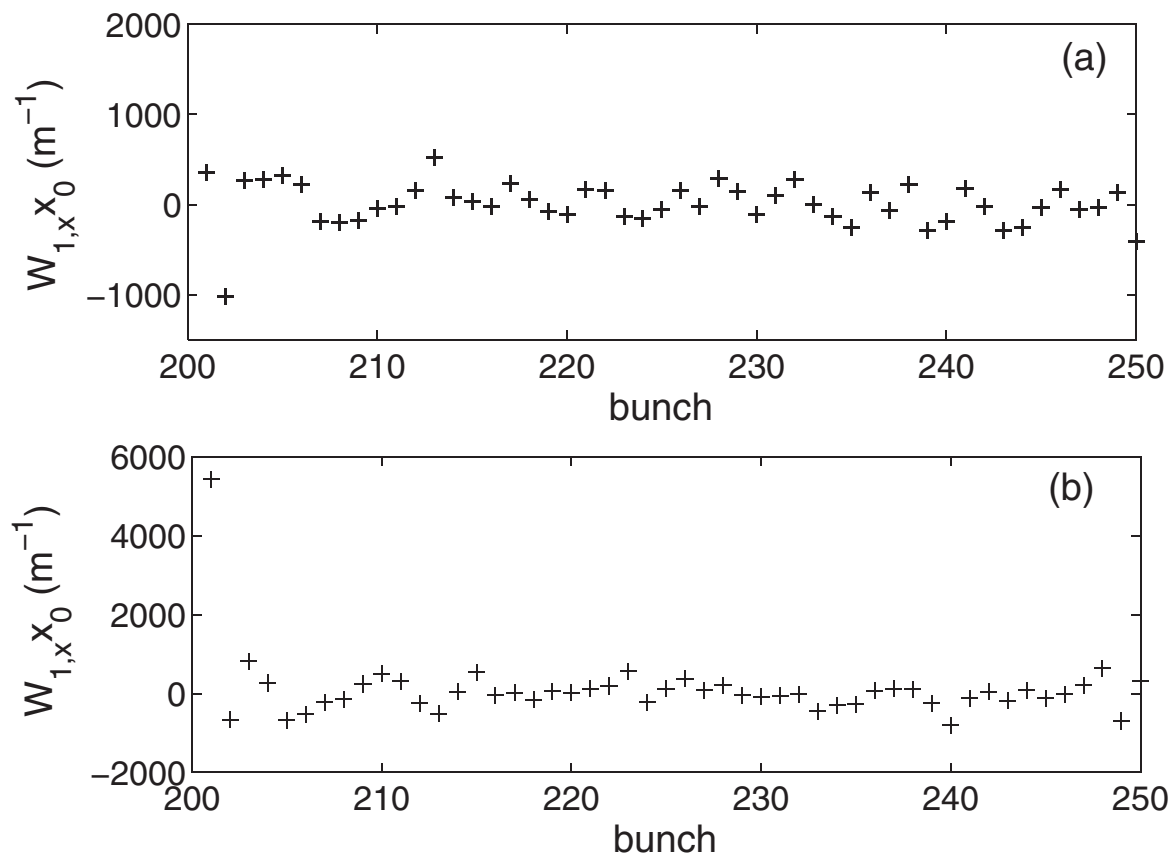

FIG. 6. Horizontal wake force for $8 \mathrm{~ns}$ spacing at KEKB. Plots (a) and (b) are given for two secondary rates, $\delta_{2, \max }=1.0$ and $\delta_{2, \max }=1.5$, respectively. Primary electrons are produced at a photon-illuminated position, and $30 \%$ of them are produced uniformly on the chamber surface. 

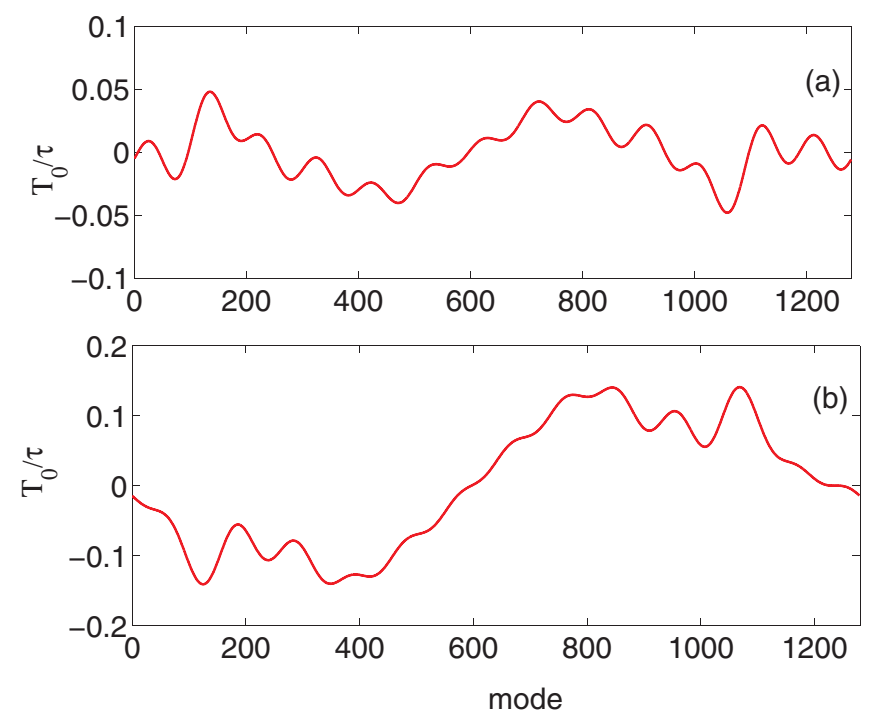

FIG. 8. (Color) Growth rate of horizontal coupled-bunch instability estimated by the wake force in Fig. 6. Plots (a) and (b) depict the growth rates for two secondary rates of $\delta_{2, \max }=1.0$ and 1.5, respectively. Primary electrons are produced at a photon-illuminated position, and $30 \%$ of them are produced uniformly on the chamber surface.

shows the growth rate per revolution for each coupledbunch mode for the vertical instability. The growth rates for 2 and $4 \mathrm{~ns}$ spacing are slightly higher than that for $8 \mathrm{~ns}$ spacing. For $8 \mathrm{~ns}$ spacing, the modes with $m=800-1000$ are the most unstable. The growth time, which is about 15 turns or faster in every case, is very rapid. For the high secondary rate, $\delta_{2 \max }=1.5$, the growth rate increases twofold though the mode spectrum shows little change.

The growth rate of the horizontal mode is shown in Fig. 8. The two plots correspond to the wake forces shown in Fig. 6. Plots (a) and (b) show the rates for two secondary rates, $\delta_{2, \max }=1.0$ and 1.5 , respectively. The modes around $m \sim 150$ are unstable in plot (a). In the unstable mode with $m+\nu_{\beta}<M / 2$, the betatron phase of each bunch advances along the bunch train ("advanced mode"). This is characteristic of the focusing wake in Fig. 6(a). For the higher secondary rate $\delta_{2, \max }=1.5$, the mode spectrum is similar to that for the vertical instability; i.e., modes with $m=800-1000$ are unstable. The mode spectrum for electrons produced uniformly is also similar to the vertical one.

\section{Wake force in a weak solenoid magnetic field}

A magnetic field affects the coupled-bunch instability through the second term of the right-hand side of Eq. (7). Effects of magnetic fields have been studied since the design stage of KEKB [20-23]. Electrons experience cyclotron motion, and the center of the cyclotron motion drifts along the chamber surface. The cyclotron frequency $\left(\omega_{c}\right)$ is $2 \pi \times 28 \mathrm{MHz}$ for $B_{z}=10 \mathrm{G}$ and linearly depends on $B_{z}$. The other frequency is obtained in the same manner as the motion in a magnetron. It is expressed by

$$
\omega_{ \pm}=\frac{\omega_{c}}{2} \pm \sqrt{\frac{\omega_{c}^{2}}{4}-\frac{r_{e} \bar{\lambda}_{p} c^{2}}{\bar{r}^{2}}},
$$

where $\bar{r}$ is the average orbit radius of the electrons. Here the radius is assumed to be larger than the cyclotron radius, and the positron beam is assumed to be a uniform charge distribution with the average line density $\bar{\lambda}_{p}$ along the longitudinal axis. If electrons are created at the chamber wall and are trapped near it by the magnetic field, the radius is close to the chamber radius $R$. If some kind of diffusion occurs in the electron motion, the radius may be smaller than the chamber radius. Of these two frequencies, one, $\left(\omega_{+}\right)$, is the cyclotron frequency slightly shifted by the beam force, and the other, $\left(\omega_{-}\right)$, which represents a spiral trajectory along the chamber surface $[21,23]$, is far slower than the cyclotron one. Perturbation of the electron motion with these frequencies is induced by a displacement of a bunch, and the coupled-bunch instability is subject to the electron motion with these frequencies. Figure 9 shows the frequencies as a function of $B_{z}$ for $\bar{r}=5,4$ and $3 \mathrm{~cm} . \omega_{-}$is faster for an electron orbit which approaches the beam position (small $\bar{r}$ ), while $\omega_{+}$is slightly slower.

Figure 10 shows the wake force for a displacement of the 600th bunch of 1,2 , and $5 \mathrm{~mm}$ at $B_{z}=10 \mathrm{G}$. Primary electrons are produced uniformly on the chamber surface and secondary ones are not considered. The results do not change for electrons produced at an illuminated position

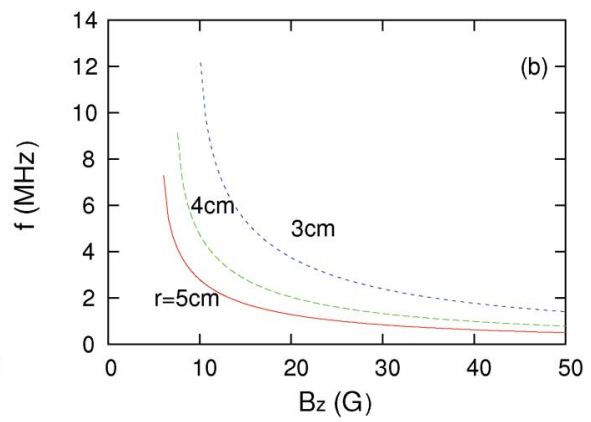

FIG. 9. (Color) Magnetron frequency as a function of $B_{z}$. Plots (a) and (b) depicts frequency $\omega_{+} / 2 \pi$ and $\omega_{-} / 2 \pi$, respectively. 

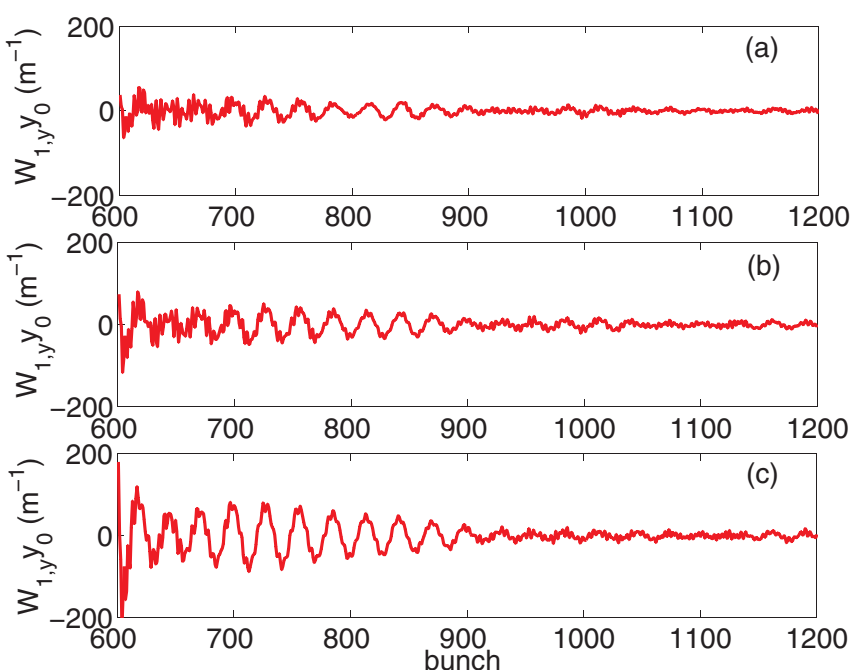

FIG. 10. (Color) Wake force for various displacements in a solenoid field, $B_{z}=10 \mathrm{G}$. Plots (a), (b), and (c) depict the wake force for displacements of the 600th of 1,2 , and $5 \mathrm{~mm}$, respectively. Primary electrons are produced uniformly on the chamber surface and secondary ones are not considered.

and for considering secondary electrons [5]. Electrons are distributed near the chamber surface in either case in an equilibrium state. The wake force has a fast frequency component corresponding to $\omega_{c}$ or $\omega_{+}$and a slow frequency component corresponding to $\omega_{-}$. The slow frequency, which is about $4 \mathrm{MHz}$ in Fig. 10, is consistent with the frequency given for the orbit radius $\bar{r}=4-5 \mathrm{~cm}$ in Fig. 9(b). We must note that the wake force with the slow frequency component is linear with the displacement, while that for the fast frequency component $\left(\omega_{c}\right)$ seen in fluctuation width of the line is constant. This fact means that the instability at the lower frequency is observed, but the instability at the higher frequency may not be observed. Another important feature of the wake force with $\omega_{-}$is the focusing nature just after the displaced bunch. Such a wake force induces advanced modes with $m+\nu_{\beta}<M / 2$, as is discussed in the horizontal instability of Fig. 8(a). Similar wake force is obtained by Wang et al. [23].

Figure 11 shows the wake force for $B_{z}=20$ and $30 \mathrm{G}$. Two frequency components are seen in both plots. Again, the fast frequency is $\omega_{+} \approx \omega_{c}$. The slow frequencies, which have 60-bunch (2 MHz) and 100-bunch $(1.25 \mathrm{MHz})$ periods for $B_{z}=20$ and $30 \mathrm{G}$, respectively, coincide with $\omega_{-}$in Fig. 9. The linearity condition of the wake force with the slow frequency was satisfied at these field strengths, but that with the fast frequency was not satisfied.

Figure 12 shows the spectra of growth modes for $B_{z}=$ 10,20 , and $30 \mathrm{G}$. To get the spectra, the wake force given by $y_{0}=5 \mathrm{~mm}$ was used. As is expected, advanced modes of the coupled-bunch instability are found, i.e., $m+$ $\operatorname{Int}\left(\nu_{y}\right)<M / 2$. For $B_{z}=10 \mathrm{G}$, the lower frequency was $6 \mathrm{MHz}$ in Fig. 10, therefore the mode with $60-43 \approx 20$
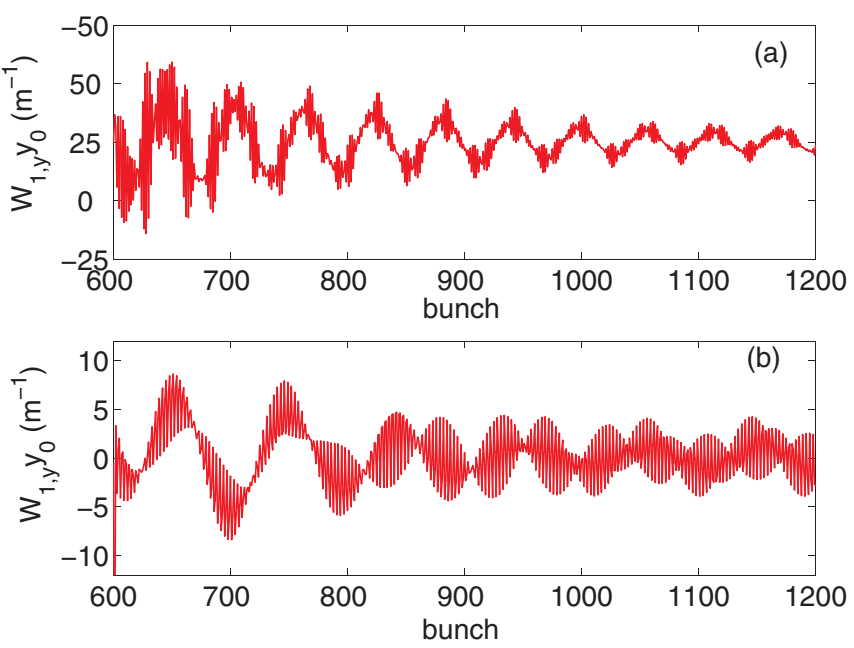

FIG. 11. (Color) Wake force for $B_{z}=20$ (a) and $30 \mathrm{G}$ (b) for a displacement of the 600th bunch of $5 \mathrm{~mm}$. Primary electrons are produced uniformly on the chamber surface and secondary ones are not considered.

appears in Fig. 12(a). Note that the revolution frequency is $0.1 \mathrm{MHz}$. For $B_{z}=20$ and $30 \mathrm{G}$, modes with $20-43+$ $1280 \approx 1260$ and $12-43+1280 \approx 1250$ appears in Figs. 12(b) and 12(c), respectively. The mode spectrum has two frequency components near the cyclotron frequency; one may be $\omega_{c}$ and the other $\omega_{+}$. However the wake force does not possess linearity for the fast frequency component. We have to wait for the results of tracking simulation to determine whether the unstable modes with $\omega_{c}$ and $\omega_{+}$are observed.

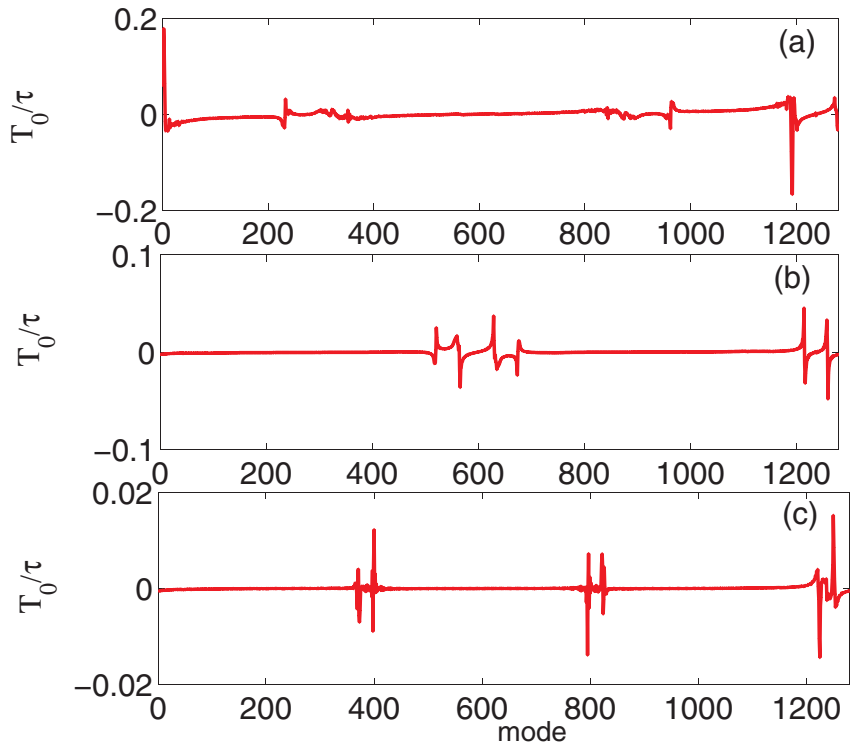

FIG. 12. (Color) Growth rate of coupled-bunch instability for $B_{z}=10,20$, and $30 \mathrm{G}$ estimated by the wake force in Figs. 10 and 11 for an initial displacement of $5 \mathrm{~mm}$. 


\section{Wake force in a strong bending magnetic field}

In a strong magnetic field, electrons move along magnetic flux lines with a circulating cyclotron motion in the plane perpendicular to the flux lines. The cyclotron frequency and radius are $\omega_{c}=2 \pi \times 19 \mathrm{GHz}$ and $r_{c}=$ $0.11 \mathrm{~mm}$ for $B \sim 1 \mathrm{~T}$ and $E=1 \mathrm{keV}$. In a bending magnet, the motion of the electrons is limited along the vertical direction. Therefore the magnetic field is approximated as $B_{y}=\infty$ for simplicity in the simulation.

Electrons emitted at the illuminated point do not contribute to the cloud buildup due to the strong bending field. Therefore, electrons which are created uniformly on the chamber surface are taken into account to estimate the wake force and instability. Though electrons move in the vertical direction only, the beam experiences not only vertical but also horizontal forces from the cloud. Since the cloud density can vary in the horizontal direction, the beam can experience a horizontal force. Figure 13 shows the horizontal and vertical wake forces due to the electron cloud in a strong bending field. A very slow frequency component with defocusing nature is seen in the horizontal wake force, while a fast focusing one is seen in the vertical wake force. Linearity of the wake force is satisfied as shown in the figure.

The mode spectrum of the growth rate is shown in Fig. 14. The growth rate is estimated for the case where the whole ring is occupied by bending magnets. For $\mathrm{KEKB}$, since the packing factor of the magnets is about $3 \%$, the growth rate is less than $3 \%$ of the value in the figure. An unstable mode with $m=1234=1280-$ $\operatorname{Int}\left(\nu_{x}\right)-1$ in plot (a) is caused by the slowest frequency
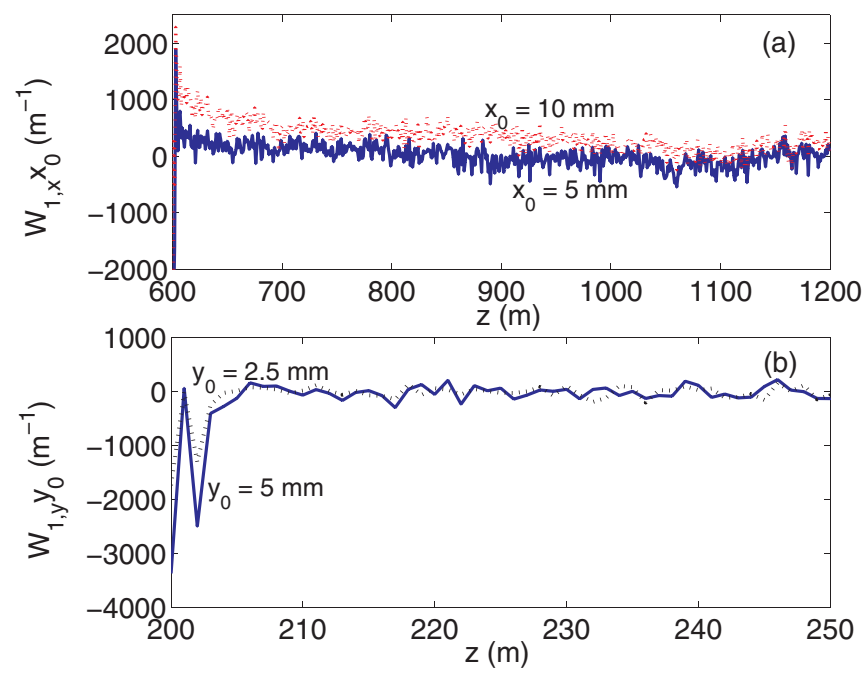

FIG. 13. (Color) Wake force due to electron cloud in a strong bending magnetic field. Plots (a) and (b) depict the horizontal and vertical wake forces for the displacement of 2.5, 5, and $10 \mathrm{~mm}$. Primary electrons are produced uniformly on the chamber surface, and secondary ones are produced with a rate of $\delta_{2, \max }=1.5$ and a reflection of $\delta_{0}=0.5$.
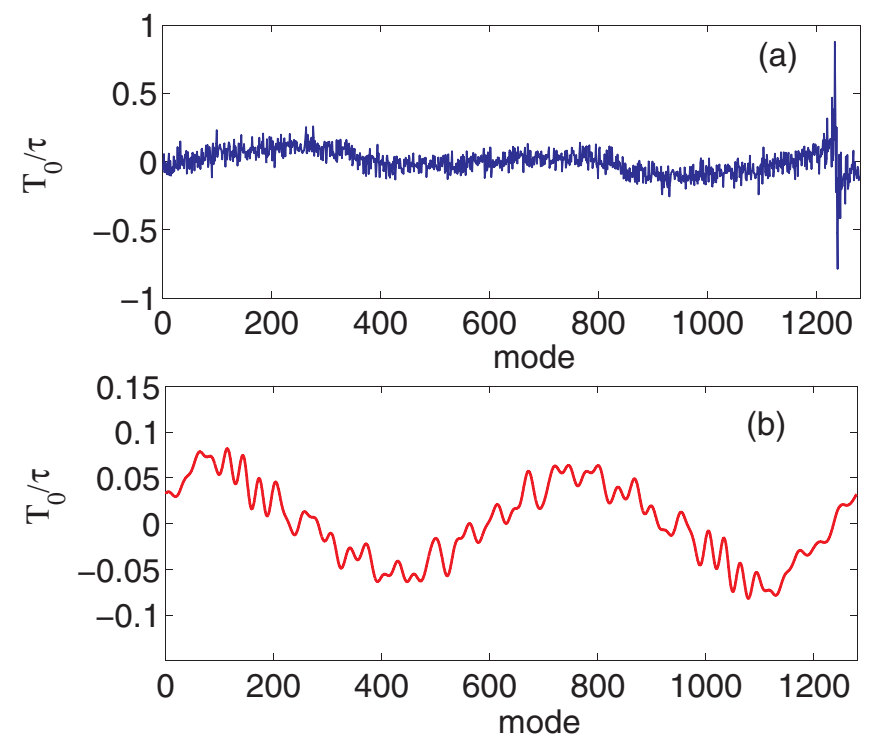

FIG. 14. (Color) Growth rate of coupled-bunch instability in bending magnet. Plots (a) and (b) depict the mode spectra of the horizontal and vertical coupled-bunch instabilities.

component of the horizontal wake force seen in Fig. 13. The vertical spectrum in plot (b) is similar to the horizontal one in Fig. 8(a). This characteristic can be explained by the planar symmetry of the electron cloud along $x$ [in drift space, Fig. 2(a)] or $y$ [in bending magnets, Fig. 2(d)].

\section{TRACKING OF THE COUPLED-BUNCH INSTABILITY}

The three equations, Eqs. (6)-(8), can be directly solved numerically $[24,25]$. The calculation time is much longer than that of the wake method. For the wake field calculation, it was sufficient to calculate the interactions of several dozen or a few hundred bunches with the electron cloud. In this tracking simulation, a thousand bunches in a ring interact with the cloud during several hundred or more revolutions, depending on the growth time. Since the calculation of the space charge field is time consuming, we use an approximation. The Poisson equation is solved only once for zero beam amplitude, that is, the space charge potential, which was calculated by the buildup simulation, is used as a constant field in this tracking simulation. This approximation is reliable and is sufficient for us, when the beam oscillation amplitude is small. At the cost of a long calculation time, the nonlinear effect and the deviation of superposition for the beam-cloud interaction are included automatically.

The transverse amplitude of each bunch is obtained as a function of time. Fourier transformation of the amplitudes of all bunches gives a spectrum of unstable modes. Experiments on coupled-bunch instabilities have been extensively performed using fast beam-position monitors $[5,26]$. The bunch centroid amplitude is directly measured by the monitors as a function of time. The mode spectrum 
given by the Fourier transformation of the experimental data can be compared with that obtained by the simulation.

\section{A. Tracking of bunches interacting with electrons in drift space}

Figure 15 shows the growth of the coupled-bunch instability given by the simulation. Electrons are produced at the illuminated point and additional 30\% of them are uniformly, and the parameters for secondary emission are $\delta_{2, \max }=1.0$ and $\delta_{0}=0.5 . M=1280$ bunches of uniform size are distributed every $8 \mathrm{~ns}$ in the simulation corresponding to a bunch frequency of $125 \mathrm{MHz}$. The evolution of maximum transverse amplitude in the bunches is shown in plot (a). The amplitude grows exponentially for far smaller values than the chamber size, while it is saturated at the order of the chamber size $(\sim \mathrm{cm})$. The growth time is about 25 turns, which is consistent with the estimate using the wake force (15 turns); see Fig. 7(c). The snapshot of the transverse position of each bunch after $150(x)$ and $100(y)$ turns is depicted in plot (b). The oscillation period varies unsteadily about 3-4 bunches for vertical and horizontal plane, respectively. The frequencies are $\approx 40 \mathrm{MHz}$, therefore the corresponding mode $(m)$ is either the advanced one with $m=400-45 \approx 350$ or the retarded one with $m=$ $1280-45-400 \approx 850$. A retarded mode is expected from the wake force in the previous section.

Figure 16 shows the mode spectrum given by Fourier transformation of the amplitude evolution. A low secondary electron rate $\delta_{2, \max }=1.0$ and $\delta_{0}=0.5$ is used in this simulation. The amplitudes between 50 through 100 turns, which are in the exponential growth regime, are used for the calculation of the mode spectrum. Plots (a) and (b) depict the horizontal and vertical spectra, respectively, for electrons emitted at the illuminated point. The fast unstable modes are distributed around 800-900 in the vertical direction. The horizontal spectrum also includes advanced
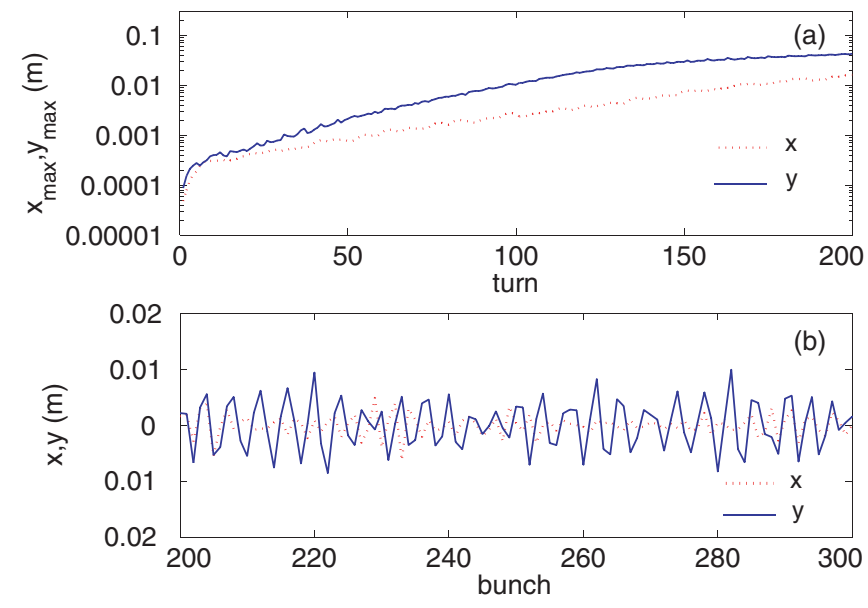

FIG. 15. (Color) Growth of the horizontal ( $x$ : solid curve) and vertical ( $y$ : dotted curve) amplitude (a) and bunch oscillation pattern (b) for bunches interacting with electrons in drift space.

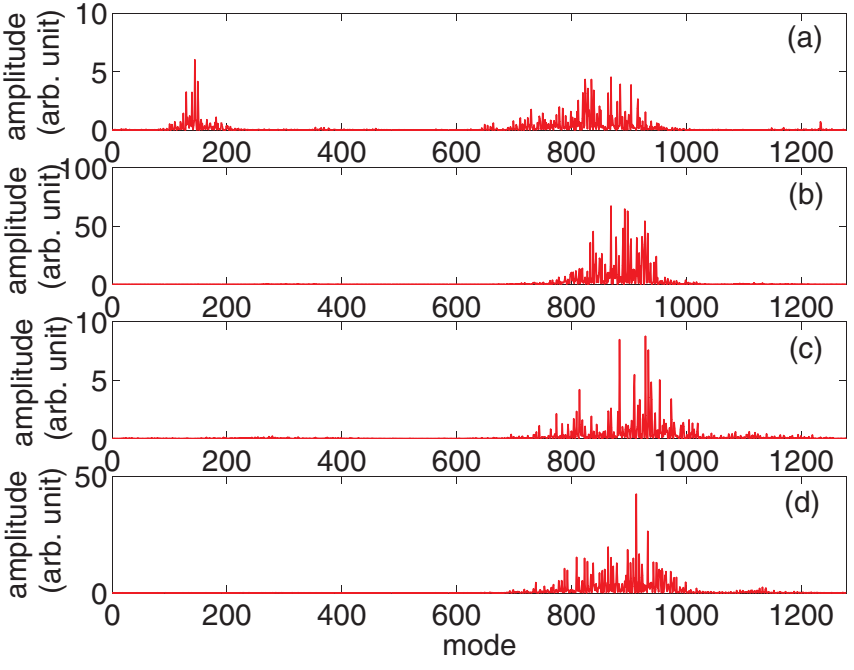

FIG. 16. (Color) Mode spectrum given by Fourier transformation of the amplitude evolution. Plots (a) and (b) depict the horizontal and vertical spectra, respectively, for electrons emitted at the illuminated point. Plots (c) and (d) depict the situation for electrons emitted uniformly. Secondary electron rate is $\delta_{2, \max }=1.0$ and a reflection of $\delta_{0}=0.5$.

modes, $m=100-200$. These pictures can be compared with Figs. 7(a) and 8(a), respectively. The mode spectra are qualitatively coincident with those given by the wake force. Plots (c) and (d) depict the situation for electrons emitted uniformly. Figure 17 shows the same situation for $\delta_{2, \max }=1.5$ and a reflection of $\delta_{0}=0.5$. Other unstable modes are now seen around 1100 .

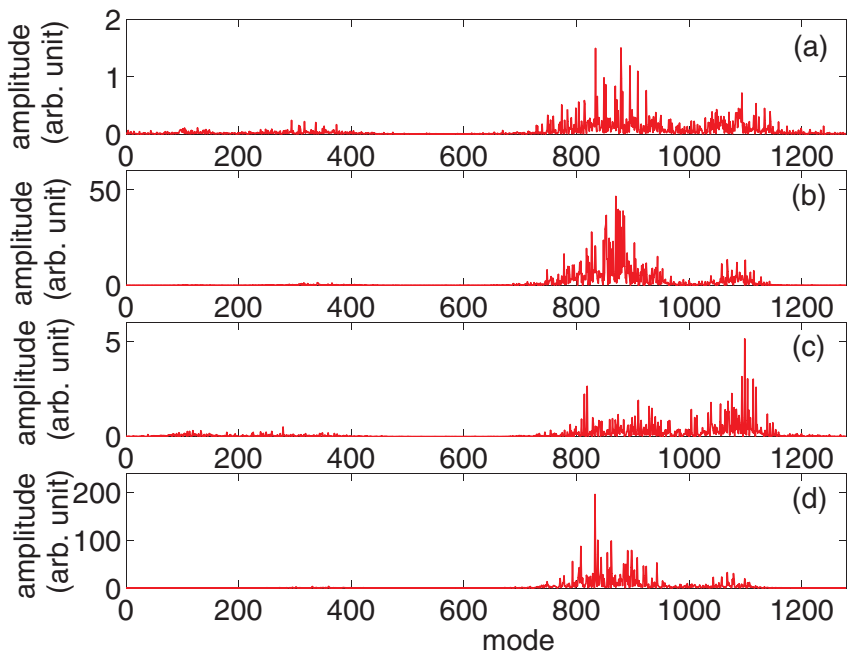

FIG. 17. (Color) Mode spectrum given by Fourier transformation of the amplitude evolution for high secondary emission rate, $\delta_{2, \max }=1.5$ and a reflection of $\delta_{0}=0.5$. Plots (a) and (b) depict the horizontal and vertical spectra, respectively, for electrons emitted at the illuminated point. Plots (c) and (d) depict the situation for electrons emitted uniformly. 

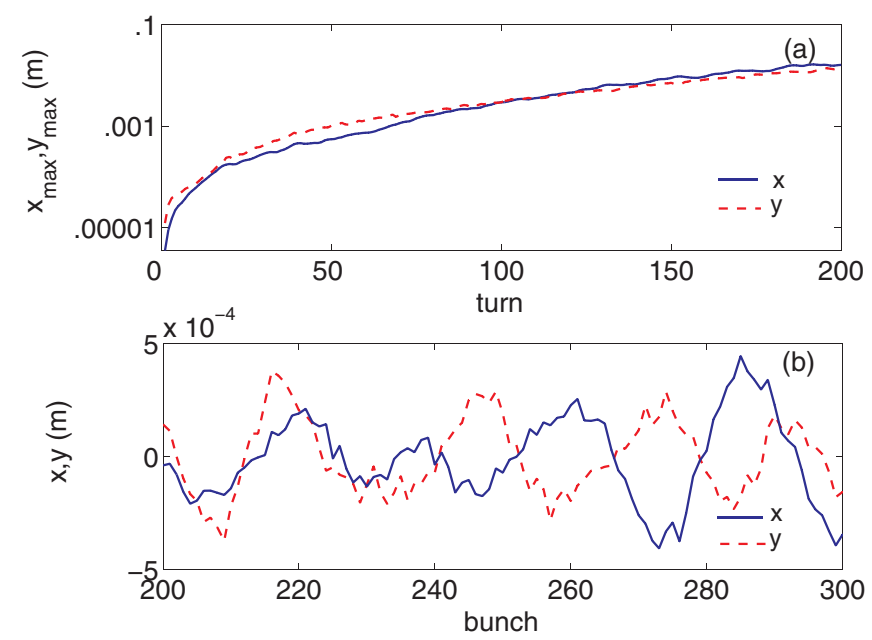

FIG. 18. (Color) Growth of the amplitude (a) and bunch oscillation pattern (b) for $B_{z}=10 \mathrm{G}$. Primary electrons are produced uniformly and secondary ones are produced with $\delta_{2, \max }=1.5$ and a reflection of $\delta_{0}=0.5$.

\section{B. Tracking in a weak solenoid magnetic field}

As is seen in the previous section, the wake force in a weak solenoid magnetic field has two frequency components, $\omega_{-}$and $\omega_{+} \approx \omega_{c}$. One component of the wake force, $\omega_{-}$, possesses linearity with respect to bunch displacement, but the other component, $\omega_{+}$, does not. The tracking simulation gives the ability to investigate whether and how modes with or without linearity are observed.

Figure 18 shows the growth of the coupled-bunch instability for $B_{z}=10 \mathrm{G}$. The evolution of the maximum transverse amplitude along the bunch train is plotted in Fig. 18(a). The amplitude grows exponentially for an amplitude $<1 \mathrm{~cm}$, and the growth time is about 40 turns.
The growth rate using the wake force in Fig. 12 was 10 turns. The incompleteness of linearity and superposition of the wake force causes the slower growth rate. The transverse amplitude of each bunch after 100 turns is depicted in Fig. 18(b). The bunch-by-bunch oscillation has a slower frequency than that with no magnetic field. The oscillation period is about 20 bunches, corresponding to a frequency of $6 \mathrm{MHz}$. The frequency is consistently seen in the wake force (Fig. 10) and $\omega_{-}$(Fig. 9). A structure with the cyclotron frequency $\omega_{c}=2 \pi \times 24 \mathrm{MHz}$, which is a 5bunch period, is seen a little but it is not clear.

Figure 19 shows the mode spectra obtained by Fourier transformation of the amplitude evolution. As is expected, the mode numbers are distributed from 0 to 50 for $B_{z}=$ $10 \mathrm{G}$ in plot (a). The modes are caused by a focusing wake force at a driving frequency $4-10 \mathrm{MHz}$, where the frequency corresponds to the low frequency $\left(\omega_{-}\right)$.

For a higher magnetic field $B_{z} \geq 20 \mathrm{G}$, very high modes are observed. As is predicted in the previous section, the modes around 1250-1260 are observed in plots (c) and (d). The modes are advanced ones, which recall the focusing wake force again, and the peak position of the mode number has shifted in the negative direction (slower frequency), having wrapped around from their location ( $0-$ 50) in plot (a) and (b). The spectrum for $B_{z}=30 \mathrm{G}$ is qualitatively similar to that for 10 and $20 \mathrm{G}$. The peak position, which is 1250 , further approaches to the slowest advanced mode $m=1235=1280-45$.

The same simulation was done for electrons produced at the illuminated position. For low field strength $B_{z} \leq 10 \mathrm{G}$, growth rate and spectrum were similar as those for electrons uniformly produced. For $B_{z} \geq 20 \mathrm{G}$, growth rate was reduced $\sim 1 / 3$ or less, probably due to the local electron distribution near the illuminated position.
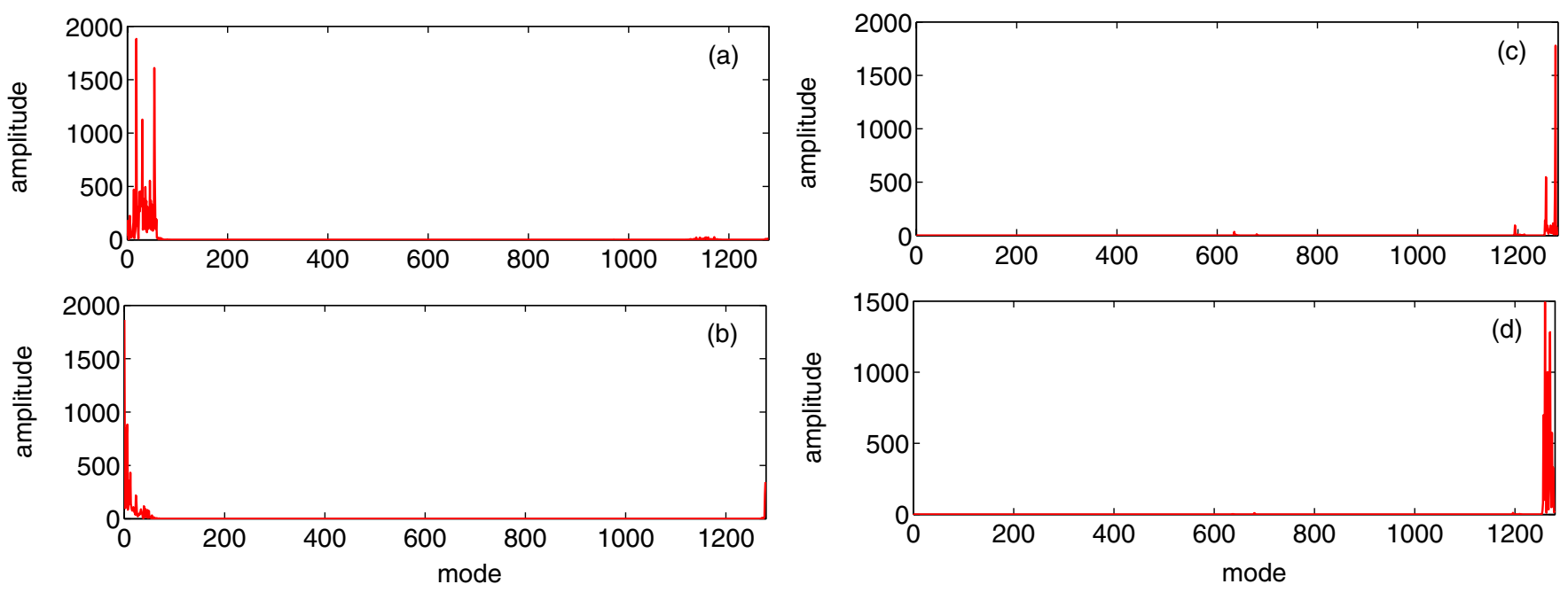

FIG. 19. (Color) Mode spectra of the coupled-bunch instability caused by an electron cloud in a weak solenoid field. Plots (a) and (b) depict the horizontal and vertical spectra for $B_{z}=10 \mathrm{G}$, respectively. Plots (c) and (d) are for $B_{z}=20 \mathrm{G}$. 


\section{Tracking in a strong bending magnetic field}

A tracking simulation was also performed for a beam interacting with electrons in a strong bending magnetic field $\left(B_{y}=\infty\right)$. The beam interacts with electrons which move along the vertical direction. A horizontal wake force at low frequency and a vertical one with fast damping (broadband) are found for an electron cloud in the field in the previous section. Figure 20 shows the growth of the maximum amplitude of the bunches and the bunch oscillation pattern. The horizontal growth is faster than the vertical growth. The growth rates are again found under the assumption that the whole ring is covered by bending magnet, and therefore the actual growth rate is slower by a factor of 20-100. The bunch oscillation pattern is a very slow wave, which is about 1 revolution period, for the horizontal motion, while the vertical oscillation has many periods.

Figure 21 shows the mode spectra given by the evolution of the bunch position. The horizontal spectrum has a sharp peak at $1234=1280-1-\operatorname{Int}\left(\nu_{x}\right)$, which corresponds to the slow retarded oscillation seen in the bunch pattern, and is consistent with the spectrum given by the wake force. The vertical spectrum has two broad peaks around 150 and 900 , which is similar to that given by the wake force. A similar spectrum is seen in horizontal direction in drift space, see Figs. 8(a) and 16(a).

\section{LONGITUDINAL COUPLED-BUNCH INSTABILITY}

A longitudinal instability was first discussed by Rumolo and Zimmermann [27] as a single-bunch effect, and then a
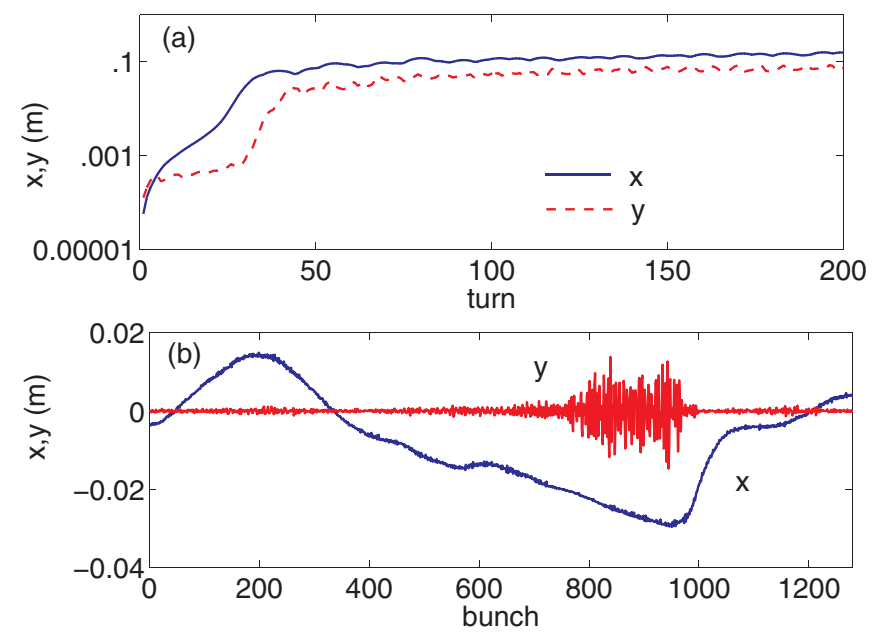

FIG. 20. (Color) Growth of the horizontal ( $x$ : solid curve) and vertical ( $y$ : dotted curve) amplitude (a) and bunch oscillation pattern (b). Primary electrons are produced uniformly and secondary ones are produced with $\delta_{2, \max }=1.5$ and a reflection of $\delta_{0}=0.5$. The bunch oscillation pattern (b) is obtained at 25th and 50th turns for horizontal and vertical motions, respectively.
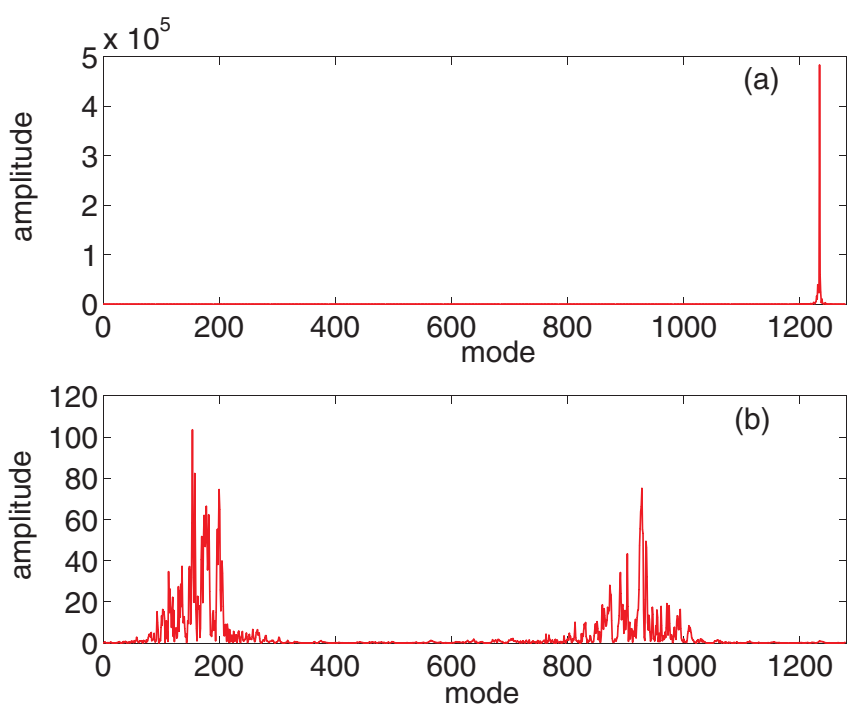

FIG. 21. (Color) Mode spectra of the coupled-bunch instability caused by an electron cloud in a strong bending magnetic field. Plots (a) and (b) show the horizontal and vertical spectra, respectively.

coupled-bunch mode of longitudinal instability was proposed by Novokhatski [10].

The longitudinal electric field at $r$ is calculated for an electron cloud with cylindrical symmetry [28].

$$
E_{z}(z, r)=Z_{0} \int_{r}^{R} j_{r}\left(r^{\prime}, z\right) d r^{\prime}
$$

where $Z_{0}$ is the vacuum impedance, $j_{r}$ the radial component of electron current in the cloud and $R$ the chamber radius. The longitudinal wake force is estimated in a similar manner as in the case of the transverse wake force: that is, it is longitudinal kicks of bunches, which are caused by a perturbation of electron cloud induced by a bunch with a longitudinal displacement $z_{0}$.

Figure 22 shows the longitudinal electric field as a function of time. $E_{z}(r=0, z)$, denoted by blue markers, is the electric field which the bunch experiences. Note that a bunch is now represented by 10 slices. Averaged longitudinal wake force of the slices is obtained as $d E_{z} / d z=$ $(10.4,14.8,18.8,3.0,1.6) \mathrm{V} / \mathrm{m}^{2}$ at bunch locations $z=$ $(-1,-2,-3,-4,-5) \times L_{\mathrm{sp}}$, where $M=5120$.

The growth rate is obtained by the dispersion relation,

$$
\begin{aligned}
\Omega_{m}-\omega_{s}= & -\frac{N_{p} r_{e} \eta c}{2 \gamma T_{0} \omega_{s}} \sum_{n=1}^{n_{0}} W_{0}^{\prime \prime}\left(-\frac{n}{M} L\right)[1 \\
& \left.-\exp \left(2 \pi i n \frac{m+\nu_{s}}{M}\right)\right],
\end{aligned}
$$

where $W_{0}^{\prime \prime}$ and $d E / d z$ are related by $d E / d z=$ $\left(N_{p} e / L\right) W_{0}^{\prime \prime}$.

Figure 22(b) informs $\Delta E \sim 1 \mathrm{~V} / \mathrm{m}$ for the longitudinal displacement of $z_{0}=10 \sigma_{z}$ during $0-6 \mathrm{nsec}$. The fastest growth rate $\operatorname{Imag}\left(\Omega_{m}\right)$ was $10 \mathrm{~s}^{-1}$ in a calculation for 

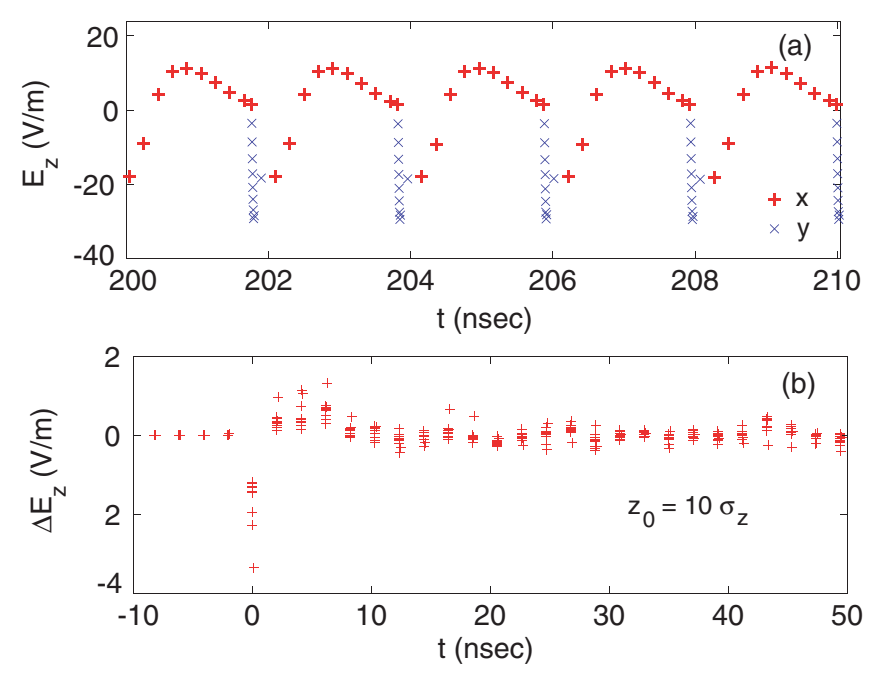

FIG. 22. (Color) Longitudinal electric field (a) and wake force (b) for super KEKB (2 ns spacing). A bunch experiences an electric field plotted by blue markers $(X)$ in plot $(a)$. The red markers ( + ) represent $E_{z}$ in the interbunch gap.

SuperKEKB $\left(N_{p}=1.17 \times 10^{11}, M=5120\right)$, other parameters in Eq. (26) are the same as those of KEKB) as shown in Fig. 23. The longitudinal coupled-bunch instability is not a serious issue in present machines.

\section{CONCLUSION}

We have studied the coupled-bunch instability caused by electron clouds. The coupled-bunch instability is caused by bunch-by-bunch correlations due to the electron cloud. The instability was analyzed using analytic, semianalytic and tracking simulation methods.

The analytic method gave the wake field as a broadband resonator and the corresponding mode spectrum for electron clouds in drift space. The resonator model with the

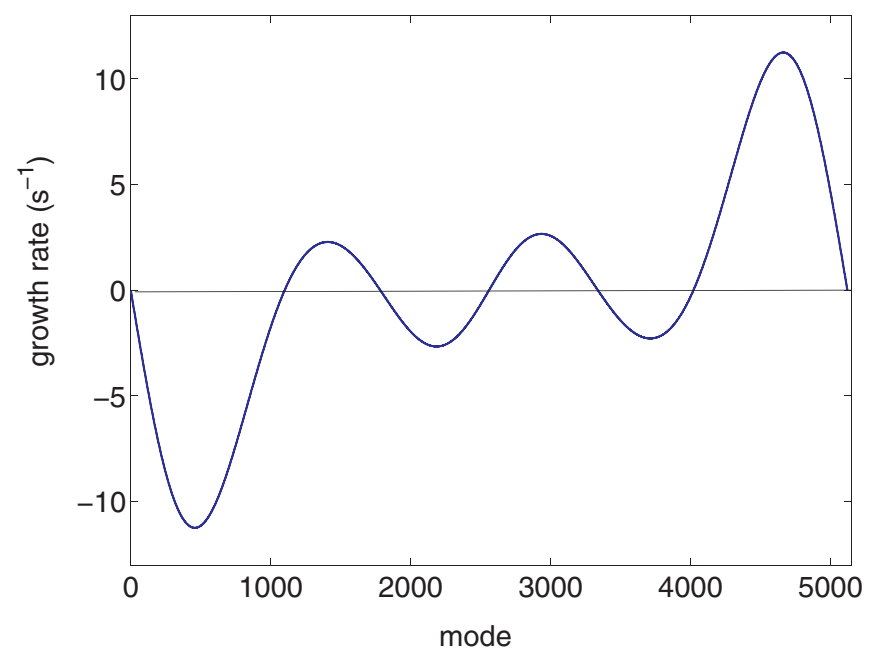

FIG. 23. (Color) Growth rate $\left(\tau^{-1}\right)$ of longitudinal coupledbunch mode $(M=5120)$. cloud size $(0.7 \mathrm{~cm} \times 0.7 \mathrm{~cm})$ and density $\left(\lambda_{e}=\right.$ $0.1 \times 10^{10} \mathrm{~m}^{-1}$ ) was consistent with those given by the semianalytical method.

In the semianalytical method, the wake force was calculated by simulation. Linearity and superposition are assumed in order to estimate the mode spectrum of the coupled-bunch instability. Wake forces for electrons in drift space, in a weak solenoid field and in a strong bending magnetic field, were evaluated. The mode spectrum of the coupled-bunch instability was estimated from the wake force. This method is practical, but is not omnipotent. For example when a magnetic field is applied, the wake force is not evidently linear with displacement amplitude; therefore this method is not useful.

The tracking simulation treats the nonlinear features and breaking of the superposition for the beam-electron cloud interaction correctly. The growth rate of each coupledbunch mode was obtained by the Fourier analysis of the dipole amplitudes of the bunches given by the tracking simulation. Comparison of the results from the semianalytic and tracking method made the nature of the coupledbunch instability clear.

The mode spectra for the growth were obtained for electrons in drift space, in a weak solenoid field and in a strong bending magnetic field. The growth rate was very rapid, $\sim 25$ turns, in drift space. When the cloud has cylindrical symmetry in the chamber, instability appears at around the $800 \sim 1100$ th mode for KEKB at 8 ns spacing and the number of bunch of $M=1280$. When the cloud has a flat distribution in the horizontal plane, the horizontal instability appears at around modes $100 \sim 200$.

When a weak solenoid field was applied, the growth rate was reduced; for example it was 40 turns for $B_{z}=10 \mathrm{G}$ in the tracking simulation. The wake force has frequency components expressed by Eq. (24). In the tracking simulation, only the slow frequency component with $\omega_{-}$was observed. This feature is reasonable, since the fast frequency component of the wake force did not possess linearity with respect to the dipole amplitude. The wake force for the lower frequency was focusing along the bunch train. In the tracking simulation, advanced modes near zero, were observed, as is expected for a focusing wake. It will be interesting to examine the focusing wake using an analytic theory, in future work.

The advanced modes were observed in experiments at the KEKB LER [5]. The measured modes were similar to those obtained for $B_{z}=10 \mathrm{G}$ in the simulation. In these experiments, a solenoid field of $B_{z}=30-50 \mathrm{G}$ was applied. This means that the effective magnetic field was smaller, or else the electrons stayed nearer the beam than our expectation, $\bar{r} \ll R$ in Eq. (24) - for example, perhaps electrons were not confined near the chamber due to some kind of diffusion.

In a strong bending magnetic field, electrons move primarily in the vertical direction. Each electron moves 
in the vertical direction but the electron cloud can have a horizontal coherent motion due to the density modulation in the horizontal direction. Therefore an electron cloud in a bending magnet induces a wake force not only in the vertical but also in the horizontal direction. Wake force analysis and tracking simulation showed instabilities with the lowest frequency mode in the horizontal direction, and with modes similar as those in the drift space in the vertical direction.

Electron clouds can cause a longitudinal coupled-bunch instability. The growth of the instability does not appear to be a serious issue in present positron storage rings.

\section{ACKNOWLEDGMENTS}

The authors acknowledge members of KEKB commissioning group. They also thank discussions with Zimmermann and Novokhatski, who motivated the study of the longitudinal instability. This work is supported by the Large Scale Simulation Program No. 123 (FY2004) of KEK.

[1] M. Izawa, Y. Sato, and T. Toyomasu, Phys. Rev. Lett. 74, 5044 (1995).

[2] K. Ohmi, Phys. Rev. Lett. 75, 1526 (1995).

[3] T. Holmquist and J. Rogers, Phys. Rev. Lett. 79, 3186 (1997).

[4] Z. Guo et al., Phys. Rev. ST Accel. Beams 5, 124403 (2002).

[5] S. S. Win, H. Fukuma, K. Ohmi, and S. Kurokawa, in Proceedings of ECLOUD'02, edited by G. Rumolo and F. Zimmermann (CERN, Geneva, 2002), pp. 199-205.

[6] H. Fukuma, J. Flanagan, K. Hosoyama, T. K. T. Ieiri, T. Kubo, M. Suetake, S. Uno, S. S. Win, and M. Yoshioka, in High Intensity and High Brightness Hadron Beams, AIP Conf. Proc. No. 642 (AIP, Geneva, 2003), pp. 357-359.

[7] K. Ohmi and F. Zimmermann, Phys. Rev. Lett. 85, 3821 (2000).

[8] F. Zimmermann, in Proceedings of ECLOUD'04, edited by M. A. Furman (CERN, Geneva, 2005).

[9] J. Flanagan, K. Ohmi, H. Fukuma, N. Hiramatsu, M. Tobiyama, and E. Perevedentsev, Phys. Rev. Lett. 94, 054801 (2005).
[10] A. Novokhatski, in Proceedings of 30th Advanced ICFA Beam Dynamics Workshop on High Luminosity $e^{+} e^{-}$ Colliders, edited by J. Seeman et al. (SLAC, Stanford, 2003).

[11] M. Bassetti and G. Erskine, CERN Technical Report No. ISR TH/80-06, 1980.

[12] M. A. Furman and G. R. Lambertson, in Proceedings of the 1997 Particle Accelerator Conference, Vancouver, edited by M. Comyn et al. (IEEE, Piscataway, 1997), pp. 1617-1620.

[13] M.A. Furman and G. R. Lambertson, in Proceedings of the International Workshop on Multibunch Instability in Future Electron and Positron Accelerator (MBI97), $K E K$, edited by Y.H. Chin (KEK, Tsukuba, 1997), pp. 190-199.

[14] V. Baglin, I. Collins, B. Henrist, N. Hilleret, and G. Vorlaufer, LHC Project Report No. 472, 2001.

[15] M. Nishiwaki and S. Kato, J. Vac. Soc. Jpn. 48, 118 (2005).

[16] Y. Suetsugu et al. [Nucl. Instrum. Methods Phys. Res., Sect. A (to be published)].

[17] S. Heifets, in Proceedings of the International Workshop on Collective Effects and Impedance for B-Factories (CEIBA95), KEK, edited by Y.H. Chin (KEK, Tsukuba, 1995), pp. 295-321.

[18] G. V. Stupakov, LHC Project Report No. 141, 1997.

[19] K. Ohmi, F. Zimmermann, and E. A. Perevedentsev, Phys. Rev. E 65, 016502 (2002).

[20] KEKB Design Group, KEK Technical Report No. 95-7, 1995.

[21] K. Ohmi and S. Matsumoto, in Proceedings of the 1998 Asian Particle Accelerator Conference, edited by Y.H. Chin et al. (KEK, Tsukuba, 1998).

[22] L. Wang, H. Fukuma, K. Ohmi, S. Kurokawa, K. Oide, and F. Zimmermann, Phys. Rev. ST Accel. Beams 5, 124402 (2002).

[23] L. Wang et al., in Proceedings of ECLOUD'04 (Ref. [8]).

[24] K. Ohmi, Phys. Rev. E 55, 7550 (1997).

[25] K. Ohmi, in Proceedings of the 1997 Particle Accelerator Conference, Vancouver, edited by M. Comyn et al. (IEEE, Piscataway, 1997), pp. 1667-1670.

[26] M. Tobiyama and E. Kikutani, Phys. Rev. ST Accel. Beams 3, 012801 (2000).

[27] G. Rumolo and F. Zimmermann, in Proceedings of ECLOUD'02 (Ref. [2]), pp. 147-154.

[28] A. Novokhatski and J. Seeman, SLAC-PUB 10327, 2004. 